\title{
PERSPECTIVES ON THE CONSTRUCTION AND COMPACTIFICATION OF MODULI SPACES
}

\author{
RADU LAZA
}

\begin{abstract}
In these notes, we introduce various approaches (GIT, Hodge theory, and KSBA) to constructing and compactifying moduli spaces. We then discuss the pros and cons for each approach, as well as some connections between them.
\end{abstract}

\section{INTRODUCTION}

A central theme in algebraic geometry is the construction of compact moduli spaces with geometric meaning. The two early successes of the moduli theory - the construction and compactification of the moduli spaces of curves $\bar{M}_{g}$ and principally polarized abelian varieties (ppavs) $\overline{\mathcal{A}}_{g}$ - are models that we try to emulate. While very few other examples are so well understood, the tools developed to study other moduli spaces have led to new developments and unexpected directions in algebraic geometry. The purpose of these notes is to review three standard approaches to constructing and compactifying moduli spaces: GIT, Hodge theory, and MMP, and to discuss various connections between them.

One of the oldest approach to moduli problems is Geometric Invariant Theory (GIT). The idea is natural: The varieties in a given class can be typically embedded into a fixed projective space. Due to the existence of the Hilbert schemes, one obtains a quasi-projective variety $X$ parametrizing embedded varieties of a certain class. Forgetting the embedding amounts to considering the quotient $X / G$ for a certain reductive algebraic group $G$. Ideally, $X / G$ would be the moduli space of varieties of the given class. Unfortunately, the naive quotient $X / G$ does not make sense; it has to be replaced by the GIT quotient $X / / G$ of Mumford [MFK94. While $X / / G$ is the correct quotient from an abstract point of view, there is a price to pay: it is typically difficult to understand which are the semistable objects (i.e. the objects parameterized by $X / / G$ ) and then some of the semistable objects are too degenerate from a moduli point of view. Nonetheless, $X / / G$ gives a projective model for a moduli space with weak modular meaning. Since the GIT model $X / / G$ is sometimes more accessible than other models, $X / / G$ can be viewed a first approximation of more desirable compactifications of the moduli space.

A different perspective on moduli is to consider the variation of the cohomology of the varieties in the given moduli stack $\mathcal{M}$. From this point of view, one considers the homogeneous space $\mathbb{D}$ that classifies the Hodge structures of a certain type, and then the quotient $\mathbb{D} / \Gamma$ which corresponds to forgetting the marking of the cohomology. The ideal situation would be a period map $\mathcal{P}: \mathcal{M} \rightarrow \mathbb{D} / \Gamma$ which is an isomorphism, or at least a birational map. Results establishing the (generic)

The author was partially supported by NSF grants DMS-1200875 and DMS-125481 (CAREER), and by a Sloan Fellowship. 
injectivity of the period map are called "Torelli theorems", and a fair number of such results are known. Unfortunately, the image of $\mathcal{P}$ in $\mathbb{D}$ is typically very hard to understand: Griffiths' transversality says that the periods of algebraic varieties vary in a constrained way, which gives a highly non-trivial systems of differential equations. Essentially, the only cases where we don't have to deal with these issues are the classical cases of ppavs and $K 3$ surfaces, for which all our knowledge on their moduli is obtained by this Hodge theoretic construction. Furthermore, having a good period map gives numerous geometric consequences. The reason for this is that the spaces $\mathbb{D} / \Gamma$ have a lot of structure that can be translated into geometric properties. While it is advantageous to get a description of the moduli space as a locally symmetric variety, in practice very few examples are known. We will briefly mention some enlargement of the applicability of period map constructions to moduli beyond ppavs and $K 3 \mathrm{~s}$. Finally, we will review some work of Looijenga which gives some comparison results for the case when both the GIT and Hodge theoretic approach are applicable. This is in some sense an ideal situation as both geometric and structural results exist.

While the first two approaches are based on considering the properties of smooth objects, and then constructing a global moduli space. The third approach takes a different tack: one constructs a moduli space by gluing local patches. This gives a moduli stack, and the main issue is to carefully choose degenerations such that one obtains a proper and separated stack. By the valuative criteria, it suffices to consider 1-parameter degenerations. From a topological point of view, the ideal model is a semistable degeneration $\mathfrak{X} / \Delta$, but then the central fiber is far from unique. A fundamental insight comes from the minimal model program (MMP): the canonical model of varieties of general type is unique. Consequently, by allowing "mild" singularities, one obtains a unique limit for a 1-parameter degeneration, leading to a proper and separated moduli stack, and (under mild assumptions) even a projective coarse moduli space. This theory was developed by Kollár-ShepherdBarron-Alexeev (KSBA) with contributions from other authors. The relationship between the KSBA approach and the other two approaches is not well understood. We briefly review some partial results on this subject. In one direction, the connection between du Bois and semi-log-canonical (slc) singularities gives a link between KSBA and Hodge theory. In a different direction, the Donaldson-Tian theory of K-stability establishes a connection between GIT and KSBA stability. These topics are rapidly evolving and suggest that much is still to be explored in moduli theory.

The overarching theme of these notes is that each approach sheds light on a different aspect of the moduli problem under consideration. By taking together different approaches one obtains a fuller picture of a moduli space and its compactifications. As examples of this principle, we point out to the theory of variation of GIT (VGIT) quotients of Thaddeus and Dolgachev-Hu, and the study of log canonical models for $\bar{M}_{g}$, the so called Hassett-Keel program.

Disclaimer. These notes reflect the interests and point of view of the author. We have tried to give a panoramic view of a number of topics in moduli theory and to point out some relevant references for further details. In particular, we point out the related surveys Laz13, Kol13a, CM12. We apologize for any omissions and inaccuracies. For instance, there is no discussion of deformation theory (Ser06, Har10] $)$, stacks ( Sta13 $)$, or the log geometry point of view $\left.\left(\mathrm{ACG}^{+} 13\right]\right)$. 
Acknowledgement. I have benefited from discussions with many people (including V. Alexeev, S. Casalaina-Martin, R. Friedman, P. Hacking, B. Hassett, E. Looijenga) over the years. I am particularly grateful to Y. Odaka and Z. Patakfalvi for some key comments on an earlier version of these notes.

\section{The Git Approach to constructing moduli spaces}

Geometric Invariant Theory (GIT) is probably the most natural and classical approach to constructing moduli spaces. In this section, we will review some of the main points of the GIT approach and survey some applications of GIT to moduli. The standard reference for GIT is Mumford et al. MFK94. Other good textbook references for GIT include: [New78], Dol03, and [Muk03]. For an extended discussion of the material included in this section see the survey [Laz13].

\subsection{Basic GIT and Moduli.}

1.1.1. Many moduli spaces are naturally realized as quotients $X / G$, where $X$ is some (quasi-)projective variety and $G$ a reductive algebraic group acting on $X$. The following results lead to presentations of moduli spaces as quotients $X / G$ :

(1) Given a class of polarized varieties, it is typically possible to give a uniform embedding result: for all $(V, L)$ in the given class, for $k$ large and divisible enough (independent of $V$ ), $L^{k}$ is very ample and embeds $V$ into a fix projective space $\mathbb{P}^{N}$ $(N=N(k)$ independent of $V)$. For example:

- (Bombieri's Theorem) For $V$ a smooth surface with $K_{V}$ big and nef, for all $k \geq 5$, the linear system $\left|k K_{V}\right|$ gives a birational morphism $V \rightarrow \bar{V} \subset \mathbb{P}^{N}$, where $\bar{V}$ is the normal surface obtained by contracting all the smooth $(-2)$ curves orthogonal to $K_{V}$. (Note, $\bar{V}$ is the canonical model of $V$ and has at worst du Val singularities.)

- (Mayer's Theorem) Let D be a big and nef divisor on a K3 surface, for all $k \geq 3$, the linear system $|k D|$ gives a birational morphism $V \rightarrow \bar{V} \subset \mathbb{P}^{N}$, where $\bar{V}$ is the normal surface obtained by contracting all the smooth $(-2)$ curves orthogonal to $D$.

While similar results for singular varieties and higher dimensions are more subtle, satisfactory (but non-effective) results exist in high generality (e.g. see Ale94, HMX13]). In other words, we can assume wlog that all varieties $V$ in a certain class are embedded in a fixed projective space $\mathbb{P}^{N}$.

(2) There exists a fine moduli space for embedded schemes $V$ in $\mathbb{P}^{N}$ with fixed numerical invariants (i.e. Hilbert polynomial $p_{V}(t)$ ): it is the Hilbert scheme $H:=$ $\operatorname{Hilb}_{p_{V}}\left(\mathbb{P}^{N}\right)$. This is a well known story (e.g. [Kol96, Ch. 1]), we only want to emphasize here the connection between flatness and the preservation of numerical invariants ([Har77, Thm. III.9.9]), and the fact that the Hilbert scheme is one of the very few instances of fine moduli spaces in algebraic geometry.

(3) To pass from the Hilbert scheme $H$ to a moduli space, there are two final steps. First, the Hilbert scheme parameterizes many objects that might have no connection to the original moduli problem (e.g. entire components of $H$ might parameterize strange non-reduced schemes). Thus, we need to restrict to the locus $X \subset H$ of "good" objects. To get a good theory, it is needed that $X$ is a locally closed subvariety of $H$ (e.g. the defining conditions for good objects are either open or closed conditions). Local closeness for the moduli functor holds quite generally, 
but sometimes it is quite subtle (e.g. Kol08). Finally, to forget the embedding $V \subset \mathbb{P}^{N}$ amounts to allowing linear changes coordinates on $\mathbb{P}^{N}$. In conclusion, by this construction, we essentially obtained a moduli space for varieties of a given class as a global quotient $X / G$, where $X \subset H$ is as above and $G=\operatorname{PGL}(N+1)$. (For technical reasons, we replace $X$ by its closure and $\operatorname{PGL}(N+1)$ by $\operatorname{SL}(N+1)$ in what follows.)

1.1.2. The naive quotient $X / G$ typically doesn't make sense. The correct solution is the GIT quotient $X / / G$. Ideally, we would like that

(a) the quotient $X / G$ gives 1-to-1 parameterization of the $G$-orbits in $X$, and

(b) $X / G$ has the structure of an algebraic variety (s.t. $X \rightarrow X / G$ is a morphism that is constant on orbits).

This is rarely possible as the example of $G=\mathbb{C}^{*}$ acting in the standard way on $\mathbb{A}^{1}=\mathbb{C}$ shows: There are two orbits, $\mathbb{A}^{1} \backslash\{0\}$ and $\{0\}$, but they cannot give two separate points in $X / G$ as this would contradict the continuity of $X \rightarrow X / G$ (N.B. $\{0\} \subset \overline{\mathbb{A}^{1} \backslash\{0\}}=\mathbb{A}^{1}$ ). The GIT solution is to relax the condition (a) and then use (b) to define a quotient in a universal categorical sense. In the affine case, $X=\operatorname{Spec} R$, it is easy to see that there is only one possible choice

$$
X / G:=\operatorname{Spec} R^{G},
$$

where $R^{G}$ is a ring of $G$-invariant regular functions (automatically a finitely generated algebra if $G$ is reductive). With this definition, all the expected properties of $X \rightarrow X / G$ hold except for (a), which is replaced by

(a') every point in $X / G$ corresponds to a unique closed orbit (and two orbits map to the same point in $X / G$ iff the intersection of their closures is nonempty).

For example, the invariant ring $\mathbb{C}[x]^{\mathbb{C}^{*}}$ (with $t \in \mathbb{C}^{*}$ acting by $x \rightarrow t x$ ) is the constants $\mathbb{C}$ and thus $\mathbb{A}^{1} / \mathbb{C}^{*}=\{*\}$ corresponding to two different orbits, one of which (i.e. $\{0\}$ ) is closed.

In general, a quotient $X / G$ can be constructed by gluing quotients of open affine $G$-invariant neighborhoods of points in $X$. For simplicity, we restrict here to the case of $X$ being a projective variety with an ample $G$-linearized line bundle $\mathcal{L}$. In this situation, the correct quotient from the point of algebraic geometry is

$$
X / / G:=\operatorname{Proj} R(X, \mathcal{L})^{G},
$$

where $R(X, \mathcal{L})=\oplus_{n} H^{0}\left(X, \mathcal{L}^{n}\right)$. Note that in the projective situation, the natural map $X \rightarrow X / / G$ is only a rational map: it is defined only for semistable points $x \in X^{s s}$, i.e. points for which there exists an invariant section $\sigma \in H^{0}\left(X, \mathcal{L}^{n}\right)$ non-vanishing at $x$. The stable locus $X^{s} \subseteq X^{s s}$ is the (open) set of semistable points $x$ for which the orbit $G \cdot x$ is closed in $X^{s s}$ and the stabilizer $G_{x}$ is finite. The quotient $X^{s} / G$ is a geometric quotient, i.e. satisfies both conditions (a) and (b), and thus a good outcome for a moduli problem.

At this point, we already see some issues with constructing a moduli space via GIT. First, the set of semistable points is somewhat mysterious and might not be what is expected. Secondly, if there exist strictly semistable points (i.e. $X^{s s} \backslash X^{s} \neq$ $\emptyset)$, then several orbits will correspond to the same point in the quotient $X / / G$. Thus, usually, the GIT quotients are not "modular" at the boundary. 
Remark 1.2. It is well known that it is essential to work with reductive groups $G$, in order to obtain finitely generated rings of invariants $R^{G}$. However, there exist natural situations when $G$ is not reductive. We point to Kir09 for some techniques to handle these cases. For some concrete examples of non-standard GIT (e.g. $G$ non-reductive or non-ample linearization $\mathcal{L}$ ) see CMJL12a, CMJL12b.

1.1.3. The GIT quotient $X / / G$ depends on the choice of linearization. This gives flexibility to the GIT construction, which is sometimes very useful. By definition (cf. (1.1), the GIT quotient depends on the choice of linearization $\mathcal{L}$, so it is more appropriate to write $X / /{ }_{\mathcal{L}} G$. A surprising fact discovered by Dolgachev-Hu DH98] and Thaddeus [Tha96] is that the dependence on $\mathcal{L}$ is very well behaved (see Laz13. $\S 3]$ for further discussion):

(1) There are finitely many possibilities for the GIT quotients $X / /{ }_{\mathcal{L}} G$ as one varies the linearization $\mathcal{L}$. The set of linearizations is partitioned into rational polyhedral chambers parameterizing GIT equivalent linearizations.

(2) The semistable loci satisfy a semi-continuity property. This property induces morphisms between quotients for nearby linearizations.

(3) The birational change of the GIT quotient as the linearization moves from one chamber to another by passing a wall is flip like, and can be described quite explicitly.

The above properties lead to one of main strengths of the theory of Variation of GIT quotients (VGIT): it might be possible to interpolate from an easily understood space $\mathcal{M}_{0}$ to a geometrically relevant space $\mathcal{M}_{1}$ by varying the linearization in a VGIT set-up. A spectacular application of this principle is Thaddeus' work Tha94] on Verlinde formula. A more modest application (but closer to the spirit of these notes) of VGIT to moduli is Theorem 1.8 below.

Example 1.3. We discuss here a simple example of VGIT (for details see Laz13, $\S 3.4])$ which illustrates the interpolation between stability conditions as the linearization varies. Specifically, we consider GIT for pairs $(C, L)$ consisting of a plane cubic and a line. In this situation, the $G$-linearizations are parameterized by $t \in \mathbb{Q}$, and they are effective (i.e. there exist invariant sections) for $t \in\left[0, \frac{3}{2}\right]$. The GIT stability for the pair $(C, L)$ for parameter $t \in\left[0, \frac{3}{2}\right]$ is described as follows: If $L$ passes through a singular point of $C$, then the pair $(C, L)$ is $t$-unstable for all $t>0$. Otherwise, $(C, L)$ is $t$-(semi)stable for an interval $t \in(\alpha, \beta)$ (resp. $t \in[\alpha, \beta])$, where

$$
\alpha=\left\{\begin{array}{ll}
0 & \text { if } C \text { has at worst nodes } \\
\frac{3}{5} & \text { if } C \text { has an } A_{2} \text { singularity } \\
1 & \text { if } C \text { has an } A_{3} \text { singularity } \\
\frac{3}{2} & \text { if } C \text { has a } D_{4} \text { singularity }
\end{array} \text { and } \beta=\left\{\begin{array}{ll}
\frac{3}{5} & \text { if } L \text { is inflectional to } C \\
1 & \text { if } L \text { is tangent to } C \\
\frac{3}{2} & \text { if } L \text { is transversal to } C
\end{array} .\right.\right.
$$

In other words, for $t=0$ the semistability of $(C, L)$ is equivalent to the semistability of $C$; for $t=\frac{3}{2}$ the semistability of $(C, L)$ is equivalent to the semistability of $C \cap L \subset L \cong \mathbb{P}^{1}$. The intermediate stability conditions are interpolations of these two extremal conditions.

1.1.4. Some of the main tools of GIT are: the numerical criterion, Luna's slice theorem, and Kirwan's desingularization. In general, it is very hard to describe the (semi)stability conditions for a GIT quotient $X / / G$. Essentially, the only effective 
tool for this is the numerical criterion. The main points that lead to the numerical criterion are as follows: By definition, the (semi)stability of $x \in X$ is related to the study of orbit closures. By the valuative criterion of properness, to test that an orbit is closed it suffices to consider 1-parameter families with all fibers in the same orbit. The key point now is that, in the GIT situation, it suffices to study to study 1-parameter families induced by 1-parameter subgroups $\lambda(t), t \in \mathbb{C}^{*}$. To such a family, one associates a numerical function $\mu^{\mathcal{L}}(x, \lambda)$ (where $\mathcal{L}$ is a $G$-linearization on $X$ ) which measures the limiting behavior as $t \rightarrow 0$. This leads to the following:

Theorem 1.4 (Hilbert-Mumford Numerical Criterion). Let $\mathcal{L}$ be an ample $G$ linearized line bundle. Then $x \in X$ is stable (resp. semistable) with respect to $\mathcal{L}$ if and only if $\mu^{\mathcal{L}}(x, \lambda)>0$ (resp. $\mu^{\mathcal{L}}(x, \lambda) \geq 0$ ) for every nontrivial 1-PS $\lambda$ of $G$.

The numerical criterion splits the a priori intractable problem of deciding semistability (i.e. finding non-vanishing $G$-invariant sections $\sigma$ at $x \in X$ ) into two somewhat accessible steps. The first step is a purely combinatorial step involving the weights of the maximal torus in $G$ on a certain representation. Furthermore, it is possible to include the variation of linearization in this combinatorial analysis. This first step can be effectively solved with the help of a computer (see [Laz13. $\S 4.1])$. The second step consists in interpreting geometrically the results of the first step. This is a case by case delicate analysis, and the true bottleneck for the wide applicability of GIT. In any case, for any concrete GIT problem, the numerical criterion gives an algorithmic approach to semistability (see $\$ 1.2 .1$ for a survey of the known GIT examples). In fact, it is reasonable to say that GIT is the most accessible/computable approach to a moduli spaces.

By construction the quotient $X / /{ }_{\mathcal{L}} G$ is a normal projective variety. It is natural to ask about its local structure. Of course, the local structure at an orbit $G \cdot x \in X / / G$ depends on the local structure of $x \in X$ and the stabilizer $G_{x}$. For instance if $X$ is smooth, then the geometric quotient $X^{s} / G$ has only finite quotient singularities (or equivalently, it is a smooth Deligne-Mumford stack) and thus it is well behaved. In general, for $x \in X^{s s} \backslash X^{s}$ (wlog assume $G \cdot x$ is a closed orbit, and thus $G_{x}$ reductive by Matsushima criterion) Luna's theorem gives a precise description of the local structure of the quotient near the orbit $G \cdot x \in X / / G$ :

Theorem 1.5 (Luna Slice Theorem). Given $x \in X^{s s}$ with closed orbit $G \cdot x$ and $X$ smooth, there exits a $G_{x}$-invariant normal slice $V_{x} \subset X^{\text {ss }}$ (smooth and affine) to $G \cdot x$ such that we have the following commutative diagram with Cartesian squares:

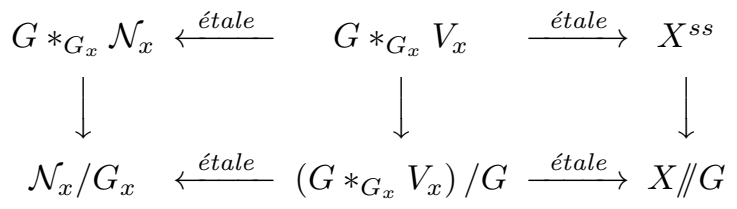

where $\mathcal{N}_{x}$ is the fiber at $x$ of the normal bundle to the orbit $G \cdot x$.

The main point here is that this theorem reduces (locally) the GIT quotient $X / / G$ to a GIT quotient by a smaller reductive group $G_{x} \subset G$ (typically $G_{x}$ is a torus, or even $\left.\mathbb{C}^{*}\right)$. As an application of this, it is easy to resolve the GIT quotients $X / / G$. Specifically, one orders the stabilizers $G_{x}$ in the obvious way, and then blows-up the strata corresponding to the maximal stabilizers $G_{x}$. Roughly, (locally) this corresponds to the blow-up of the origin in $\mathcal{N}_{x}$. Since the action 
of $G_{x}$ is distributed on the exceptional divisor of $\mathrm{Bl}_{0} \mathcal{N}_{x}$, one sees that after such a blow-up the resulting stabilizers are strictly contained in $G_{x}$. Repeating the process inductively, one arrives to the ideal situation when all the stabilizers are finite. This resolution process was developed by Kirwan Kir85. As an application, Kirwan used this desingularization process to compute the cohomology of several moduli spaces (e.g. [Kir84]). As an aside, we note that Luna's theorem and Kirwan desingularization procedure explain why various moduli spaces are nested. For instance, the exceptional divisor obtained by resolving the "worst" singularity for the GIT quotient for cubic fourfolds ( Laz09]) is naturally identified with the moduli of degree $2 K 3$ surfaces (this is also related to the discussion of $\$ 2.3$.

Remark 1.6. For a survey of the singularities of GIT quotients see [Laz13. §5.1]. For a discussion of Luna's theorem in connection to VGIT see [Laz13, §4.2].

1.1.5. The main advantage of GIT is that it gives projective models for moduli spaces with weak modular meaning. Specifically, by construction a GIT quotient $X / / G$ is a normal projective variety (assuming, as above, $X$ normal projective). Furthermore, each point of the quotient $X / / \mathcal{L}_{\mathcal{L}} G$ corresponds to a unique closed orbit. If there are no strictly semistable points, then $X / / G$ is also a geometric quotient, and thus the coarse moduli space of a proper Deligne-Mumford moduli stack. This is typically not the case: there exist strictly semistable points, and thus multiple orbits correspond to the same point in the quotient $X / / G$. It is (typically) not possible to define a functor that selects only the closed orbits. Nonetheless, the weak modular properties of GIT quotients might be the best that one can expect short of a DM stack (see Alper Alp08 for a formalization of "good" moduli stacks). Note also, the following (a consequence of the properness of $X / / G$ and of the 1-to-1 correspondence of the points in $X / / G$ with the closed orbits):

Proposition 1.7 (GIT Semistable replacement lemma). Let $S=\operatorname{Spec} R$ and $S^{*}=$ $\operatorname{Spec}(K)$, where $R$ is a DVR with field of fractions $K$ and closed point $o$. Assume that $S^{*} \rightarrow X^{s} / G$ for some GIT quotient. Then, after a finite base change $S^{\prime} \rightarrow S$ (ramified only at the special point o), there exists a lift $\tilde{f}: S^{\prime} \rightarrow X^{\text {ss }}$ of $f$ as in the diagram:

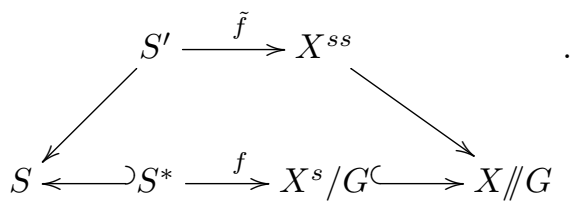

Furthermore, one can assume that $\tilde{f}(o)$ belongs to a closed orbit.

In other words, while a GIT quotient typically fails to have a modular meaning at the boundary, one can use this lemma to understand the degenerations of smooth objects and then construct or understand a good compactification of the moduli space. A concrete application of this principle is discussed in $\$ 2.3$ below. For some further discussion and some examples see [CM12, Ch. 11].

1.1.6. Unfortunately, GIT only sees "linear" features of the parameterized varieties. Consequently, quite degenerate objects might be semistable, leading to bad singularities for the GIT quotient $X / / G$ and the failure of modularity. We have mentioned several drawbacks of the GIT approach to moduli spaces: It is difficult to decide stability of objects. Also, typically there exist strictly semistable points. At these 
points, the GIT quotient is quite singular, and fails to be modular. The hidden geometric reason for these issues is that GIT only tests for linear features of the objects under consideration (e.g. see Remark 3.6). By considering asymptotic GIT (higher and higher embeddings of a given object) more of the geometric features of the varieties will be visible by means of "linear tests". Unfortunately, as discussed in $\$ 3.3$, the asymptotic GIT approach is not well behaved/well understood.

The main point we want to emphasize here is that the unstable objects always satisfy some special conditions with respect to a flag of linear subspaces (e.g. an unstable hypersurface will always contain a singular point and there will be a special tangent direction through this point). This follows for instance from the work of Kempf [Kem78]. Namely, for unstable points, there is a distinguished 1-PS $\lambda$ that destabilizes $x$ (essentially minimizing $\mu(x, \lambda) /|\lambda|$ ). Then, $\lambda$ determines a parabolic subgroup $P_{\lambda}$, which in turn is equivalent to a (partial) flag. The failure of stability involves some special geometric properties with respect to this flag. Consequently, objects that behave well with respect to linear subspaces will tend to be semistable. For instance a conic of multiplicity $\frac{d}{2}$ is semistable when viewed as a curve of degree $d$. Of course, such objects would be disallowed by other more "modular" approaches. For instance, for $d=4$ (plane quartics, or genus 3 curves), the double conic should be replaced by hyperelliptic curves.

1.2. Applications of GIT to moduli. In this section, we briefly review the scope of GIT constructions in moduli theory.

1.2.1. Survey of GIT constructions in moduli theory. GIT and moduli were tightly connected for over a hundred years. Initially, in late 1800s/early 1900s, the focus was on computing explicitly the rings of invariants for various quotients $X / G$ (for example the ring of invariant polynomials for cubic surfaces). After Hilbert's proof of the finite generation of the ring of invariants $R^{G}$, the search of explicit invariants fell out of favor. Mumford MFK94] revived GIT to show that the moduli space of curves $M_{g}$ is quasi-projective ([MFK94, Thm. 7.13]). The case of abelian varieties (with level structure) is also discussed in Mumford's monograph (MFK94, Thm. 7.9]). A little later, Mumford and Gieseker (Mum77) proved, via GIT, that the coarse moduli space $\bar{M}_{g}$ associated to the Deligne-Mumford compactification of the moduli space of genus $g$ curves is a projective compactification of $M_{g}$ (for a discussion of this and related constructions, see the survey Mor09]). Some other major results around the same time include the proof of quasi-projectivity for the moduli of surfaces of general type (Gieseker Gie77a]) and compactifications for the moduli spaces of vector bundles over curves (Mumford, Narasimhan, Seshadri, e.g. [Ses67]) and surfaces (Gieseker Gie77b]). More recently, Viehweg Vie95 proved the quasi-projectivity of moduli of varieties of general type (see also 3.4 and 3.5 below) by using non-standard linearizations on the moduli space.

The GIT constructions for $\bar{M}_{g}$ or moduli of surfaces of general type involve asymptotic GIT, i.e. given a class of polarized varieties $(V, L)$ one considers the GIT quotient of the Chow variety $\mathrm{Chow}_{k}$ (or Hilbert scheme) for higher and higher embeddings $V \rightarrow \mathbb{P}^{N(k)}$ given by $L^{k}$ (for $k \gg 0$ ). In the case of curves the quotients Chow $_{k} / / \operatorname{SL}\left(N_{k}\right)$ stabilize and give $\bar{M}_{g}$ (in fact $k \geq 5$ it is enough). For surfaces, as discussed in $\$ 3.3$, there is no stabilization for the asymptotic GIT and it is unclear how to use this asymptotic approach to construct a compact moduli space. 
In recent years, in connection to the Hassett-Keel program, there is a renewed interest in understanding non-asymptotic GIT models for $\bar{M}_{g}$. Other non-asymptotic GIT quotients that were studied include moduli for some hypersurfaces: plane sextics ([Sha80]), quartic surfaces ([Sha81a]), cubic threefolds ([All03]), cubic fourfolds ([Laz09]), and some complete intersections (e.g. AM99, CMJL12b, and Ben11]). We emphasize that for hypersurfaces it is possible to give in an algorithmic way the shape of equations defining unstable hypersurfaces. However, it is difficult to interpret geometrically the stability conditions. In fact, the higher the degree the worst the singularities that are allowed for stable objects. Consequently, GIT will give a somewhat random compactification for the moduli of hypersurfaces.

1.2.2. GIT and the Hassett-Keel program. It is of fundamental interest to understand the birational geometry of $\bar{M}_{g}$ (see the survey [Far09]), in particular the canonical model $\bar{M}_{g}^{\text {can }}$ (for $g \geq 24$ ). A fundamental insight (due to Hassett and Keel) says that one can approach this problem via interpolation (see esp. [HH09, HH13]). Namely, one defines

$$
\bar{M}_{g}(\alpha)=\operatorname{Proj}\left(R\left(\bar{M}_{g}, K_{\bar{M}_{g}}+\alpha \Delta\right)\right),
$$

where $\Delta$ is the boundary divisor in $\bar{M}_{g}$. For $\alpha=1$ (and all $g$ ) one gets the DeligneMumford model $\bar{M}_{g}$, while for $g \geq 24$ and $\alpha=0$ one gets $\bar{M}_{g}^{c a n}$. It was observed that some of the $\bar{M}_{g}(\alpha)$ models have modular meaning. For instance, $\bar{M}_{g}\left(\frac{9}{11}\right)$ is a moduli of pseudo-stable curves, i.e. curves with nodes and cusps and without elliptic tails. It is conjectured that all $\bar{M}_{g}(\alpha)$ for $\alpha \in[0,1]$ have some (weak) modular meaning, and that they behave similarly to the spaces in a VGIT set-up. In other words, (conjecturally) via a finite number of explicit and geometrically meaningful modifications one can pass from $\bar{M}_{g}=\bar{M}_{g}(1)$ to $\bar{M}_{g}^{c a n}=\bar{M}_{g}(0)$. In this way, one would obtain a satisfactory description of the canonical model $\bar{M}_{g}^{\text {can }}$ as well as a wealth of information on the birational geometry of $\bar{M}_{g}$.

Most of the constructed $\bar{M}_{g}(\alpha)$ spaces were obtained via GIT (see [FS13a] and AH12 for some recent surveys). Roughly speaking, GIT tends to give compactifications with small boundary. Consequently, once a GIT model $\bar{M}_{g}^{G I T}$ with the correct polarization was constructed, one can show that it agrees with $\bar{M}_{g}(\alpha)$ on open subsets with high codimension boundaries in $\bar{M}_{g}(\alpha)$ and $\bar{M}_{g}^{G I T}$ respectively (and thus $\bar{M}_{g}(\alpha)$ and $\bar{M}_{g}^{G I T}$ agree everywhere). The important point here is that $\bar{M}_{g}^{G I T}$ is a projective variety and that its polarization can be easily understood by descent from a parameter space (typically a Chow variety or Hilbert scheme for small embeddings of genus $g$ curves). Another important point is that $\bar{M}_{g}^{G I T}$ comes with a (weak) modular interpretation and thus it induces such a modular interpretation for $\bar{M}_{g}(\alpha)$.

As already mentioned, the behavior of $\bar{M}_{g}(\alpha)$ seems to be parallel to that of quotients in a VGIT set-up. One might conjecture that there is a master VGIT problem modeling all of $\bar{M}_{g}(\alpha)$. Unfortunately, the best result so far is:

Theorem 1.8 ([CMJL12b]). For $\alpha \leq \frac{5}{9}$, the log minimal models $\bar{M}_{4}(\alpha)$ arise as GIT quotients of the parameter space $\mathbb{P} E$ for $(2,3)$ complete intersections in $\mathbb{P}^{3}$. Moreover, the VGIT problem gives us the following diagram: 


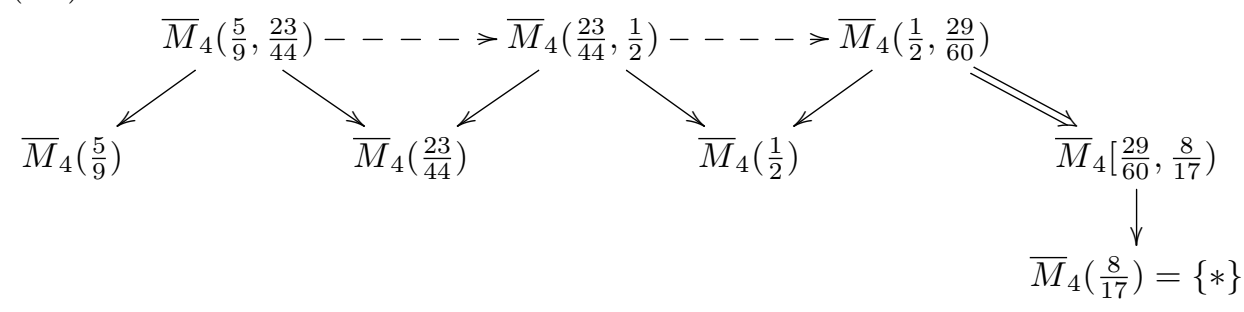

More specifically,

i) the end point $\bar{M}_{4}\left(\frac{8}{17}+\epsilon\right)$ is obtained via GIT for $(3,3)$ curves on $\mathbb{P}^{1} \times \mathbb{P}^{1}$ as discussed in Fed12;

ii) the other end point $\bar{M}_{4}\left(\frac{5}{9}\right)$ is obtained via GIT for the Chow variety of genus 4 canonical curves as discussed in CMJL12a;

iii) the remaining spaces $\bar{M}_{4}(\alpha)$ for $\alpha$ in the range $\frac{8}{17}<\alpha<\frac{5}{9}$ are obtained via appropriate $\mathrm{Hilb}_{4,1}^{m}$ quotients, with the exception of $\alpha=\frac{23}{44}$.

Remark 1.10. The Hassett-Keel program is currently established for either values of $\alpha$ close to 1 (e.g. HH09, HH13) or small values of the genus $g$ (e.g. HL10, Fed12, CMJL12a, CMJL12b], FS13b]). For some general predictions on $M_{g}(\alpha)$ see AFS10.

\section{MOdUli AND PERIODS}

A different approach to the construction of moduli spaces is based on the idea of associating to a variety its cohomology, and studying the induced variation of Hodge structures (VHS). As we will discuss here, the scope of this approach is quite limited in practice. However, when the Hodge theoretic approach is applicable, it has strong implications on the structure of the moduli space; and thus this is a highly desirable situation. We discuss some examples of moduli space for which both the GIT and Hodge theoretic approach are applicable. Each approach gives a different facet of the moduli space.

Some general references for the material discussed here include CMSP03, Voi07, GGK12, Mil13, and [SU02, Ch. 2, 3].

2.1. Period maps. The primitive cohomology of a smooth projective variety carries a polarized Hodge structure such that the associated Hodge filtration varies holomorphically in families. More formally, one says that for a smooth family $\pi: \mathcal{X} \rightarrow S$ of algebraic varieties, $\left(R^{n} \pi_{*} \underline{\mathbb{Z}}_{\mathcal{X}}\right)_{\text {prim }}$. defines a polarized Variation of Hodge Structures (VHS) over $S$, which in turn defines a period map $\mathcal{P}: S \rightarrow \mathbf{D} / \Gamma$. In order to use $\mathcal{P}$ to construct a moduli space, we need to discuss the injectivity of $\mathcal{P}$ ("Torelli Theorems") and the image of $\mathcal{P}$.

2.1.1. Period domains and period maps. The period domain $\mathbf{D}$ is the classifying space of Hodge structures of a given type. Specifically, the polarized Hodge structures of weight $n$ satisfy the Hodge-Riemman bilinear relations:

(HR1) $F^{p}=\left(F^{n-p+1}\right)^{\perp}$;

(HR2) $(-1)^{n(n-1)} i^{p-q}(\alpha, \bar{\alpha})>0$ for $\alpha \in H^{p, q}=F^{p} \cap \overline{F^{q}}$ (with $p+q=n$ ). 
The first condition (HR1) defines a projective homogeneous variety $\check{\mathbf{D}}=\mathbf{G}_{\mathbb{C}} / \mathbf{B}$, a subvariety of a flag manifold. The condition (HR2) gives that $\mathbf{D}$ is an open subset (in the classical topology) of $\check{\mathbf{D}}$, in particular a complex manifold. Note also that the period domain is homogeneous $\mathbf{D}=\mathbf{G}_{\mathbb{R}} / \mathbf{K}$ (with $\mathbf{K}=\mathbf{B} \cap \mathbf{G}_{\mathbb{R}}$ a compact subgroup of $\mathbf{G}_{\mathbb{R}}$ ) and semi-algebraic (given by algebraic inequalities involving $R e$ and Im of holomorphic coordinates). It is important to note that there are only two cases when $\mathbf{D}$ is a Hermitian symmetric domain (or equivalently $\mathbf{K}$ is a maximal compact subgroup):

- weight 1 Hodge structures (abelian variety type): $\mathbf{D}$ is the Siegel upper half space $\mathfrak{H}_{g}=\left\{A \in M_{g \times g}(\mathbb{C}) \mid A=A^{t}, \operatorname{Im}(A)>0\right\} \cong \operatorname{Sp}(2 g) / U(g)$;

- weight 2 Hodge structures with $h^{2,0}=1$ (K3 type): $\mathbf{D}$ is a Type IV domain $\left\{\omega \in \mathbb{P}\left(\Lambda_{\mathbb{C}}\right) \mid \omega \cdot \omega=0, \omega \cdot \bar{\omega}>0\right\} \cong \mathrm{SO}(2, n) / S(O(2) \times O(n))$, where $\Lambda$ is a lattice of signature $(2, k)$ (here $\check{\mathbf{D}}$ is a quadric hypersurface in $\mathbb{P}^{k+1}$; thus $\operatorname{dim} \mathbf{D}=\operatorname{dim} \check{\mathbf{D}}=k)$.

Example 2.1. For Hodge structures of Calabi-Yau threefold type with Hodge numbers $(1, h, h, 1)$, the period domain is

$$
\mathbf{D}=\operatorname{Sp}(2(1+h)) / U(1) \times U(h) .
$$

The maximal compact subgroup is $U(1+h)$ and the inclusion $U(1) \times U(h) \subset U(1+h)$ induces a natural map $\mathbf{D} \rightarrow \mathfrak{H}_{1+h}$ which is neither holomorphic or anti-holomorphic (only real analytic).

A Variation of Hodge Structure over $S$ is a triple $\left(\mathcal{V}, F^{\bullet}, \nabla\right)$ consisting of a flat vector bundle $(\mathcal{V}, \nabla)$ together with holomorphic subbundles $F^{n} \subset F^{n-1} \cdots \subset F^{0}=$ $\mathcal{V}$. We assume that the VHS is polarized (i.e. there is a compatible bilinear form such that (HR1) and (HR2) are satisfied). By passing to a trivialization of the associated local system we obtain a period map:

$$
\widetilde{P}: \widetilde{S} \rightarrow \mathbf{D},
$$

where $\widetilde{S}$ is the universal cover of $S$. Alternatively, we get

$$
\mathcal{P}: S \rightarrow \mathbf{D} / \Gamma
$$

where $\Gamma=\operatorname{Im}\left(\pi_{1}(S) \rightarrow \mathbf{G}_{Z}\right)$. Note that $\mathcal{P}$ is a locally liftable analytic map (N.B. $\mathbf{G}_{Z}$, and thus $\Gamma$, acts properly discontinuous on $\mathbf{D}$, making $\mathbf{D} / \Gamma$ an analytic space).

2.1.2. Griffiths' Transversality. Classical and semi-classical period maps. The period maps arising from algebraic geometry satisfy an additional condition: Griffiths transversality

$$
\nabla F^{p} \subseteq F^{p-1} \otimes \Omega_{S}
$$

which is a non-trivial condition except for the case when $\mathbf{D}$ is Hermitian symmetric. As noted above, there are only two cases when $\mathbf{D}$ is Hermitian symmetric: abelian varieties and $K 3$ type.

We are interested in the situation when $\mathcal{M}$ is a moduli space (stack) of smooth varieties, and

$$
\mathcal{P}: \mathcal{M} \rightarrow \mathbf{D} / \Gamma
$$

is induced by the variation of cohomology $R^{n} \pi_{*} \underline{\mathbb{Z}}$. For constructing a moduli space via periods, we would like that $\mathcal{P}$ is a birational map (a priori the period maps are analytic, but there are various algebraicity results; we ignore the issue here). 
There are two statements that one tries to prove: $\mathcal{P}$ is injective and $\mathcal{P}$ is dominant. Due to the Griffiths transversality, except for ppavs and K3 type, $\mathcal{P}$ is never dominant. Even worse, the image of the period map is typically highly transcendental. Namely, say that $Z$ (a closed analytic subvariety) is the image of a period map in $\mathbf{D}$. Then $Z \subset \mathbf{D}$ is horizontal w.r.t. the distribution given by Griffiths' transversality. Furthermore, in the algebro-geometric situation, $Z$ is stabilized by a big subgroup $\Gamma \subset G_{\mathbb{Z}}$. In fact, it is reasonable to assume $Z / \Gamma$ is quasi-projective. Then, we proved the following:

Theorem 2.2 (FL13). Let $Z$ be a closed horizontal subvariety of a classifying space $\mathbf{D}=\mathbf{G}_{\mathbb{R}} / \mathbf{K}$ for Hodge structures and let $\Gamma$ be the stabilizer of $Z$ in the appropriate arithmetic group $\mathbf{G}_{\mathbb{Z}}$. Assume that

(i) $S=\Gamma \backslash Z$ is strongly quasi-projective;

(ii) $Z$ is semi-algebraic in $\mathbf{D}$ (i.e. open in its Zariski closure in $\check{\mathbf{D}}$ ).

Then $Z$ is a Hermitian symmetric domain whose embedding in $\mathbf{D}$ is an equivariant, holomorphic, horizontal embedding.

In other words, the only cases when the image of a period map can be described purely algebraically are the Shimura type cases (see [Mil13] and [Del79]). They are slight generalizations of the classical cases of ppavs and K3 type; we call them semi-classical cases. A number of such semi-classical examples are discussed in the recent literature (see for instance the series of ball quotient examples of Kondo DK07]). These semi-classical examples are naturally understood in the context of Mumford-Tate (MT) domains GGK12. Essentially, a MT domain is the smallest homogeneous subdomains of $\mathbf{D}$ defined over $\mathbb{Q}$ which contains the image of a VHS. To belong to a MT subdomain of $\mathbf{D}$ is equivalent to saying that there exist some special Hodge tensors for the VHS. The simplest situation is that of Hodge structures with extra endomorphisms as in the following example:

Example 2.3 (Kondo Kon00]). A generic genus 3 curve $C$ is a plane quartic. To it one can associate a quartic $K 3$ surface $S$ by taking the $\mu_{4}$-cover of $\mathbb{P}^{2}$ branched along $C$. For example, if $C=V\left(f_{4}\right)$, then $S=V\left(f_{3}\left(x_{0}, x_{1} \cdot x_{2}\right)+x_{3}^{4}\right) \subset \mathbb{P}^{3}$. In this way, one gets a period map

$$
\mathcal{P}: M_{3}^{n h} \rightarrow \mathcal{F}_{4} \cong \mathcal{D} / \Gamma
$$

from the moduli of non-hyperelliptic genus 3 curves to the period domain of degree $4 K 3$ surfaces. Since the resulting $K 3$ Hodge structures are special (they have multiplication by $\mu_{4}$ ), the image of $\mathcal{P}$ will land into a MT subdomain. In this situation, the MT subdomain will be a 9-dimensional complex ball $\mathcal{B}$ embedded geodesically into the 19-dimensional Type IV domain $\mathcal{D}$. In conclusion, one gets

$$
\mathcal{P}: M_{3}^{n h} \rightarrow \mathcal{B} / \Gamma^{\prime} \subset \mathcal{D} / \Gamma,
$$

which turns out to be birational (see [Loo0] and $\$ 2.3$. This construction is also relevant to the Hassett-Keel program in genus 3 (e.g. [HL10]).

We emphasize that the situation covered by Theorem 2.2 is very special. For instance, while there exist moduli of Calabi-Yau threefolds that have period maps to Hermitian symmetric domains as in the theorem (e.g. the Borcea-Voisin Bor97. and Rohde-van Geemen Roh09 examples), most moduli of Calabi-Yau threefolds (e.g. for quintic threefolds) are not of this type (see [FL13]). 
2.1.3. Torelli theorems. The differential of a period map $\mathcal{P}: S \rightarrow \mathbf{D} / \Gamma$ for a family of algebraic varieties has a simple description in cohomological terms. Namely, since the tangent space to a Grassmannian at the point $F \subset H_{\mathbb{C}}$ is canonically $\operatorname{Hom}\left(F, H_{\mathbb{C}} / F\right)$, it follows that the tangent space to a period domain satisfies $T_{o} \mathbf{D} \subseteq \oplus_{p} \operatorname{Hom}\left(F^{p}, H_{\mathbb{C}} / F^{p}\right)$. Due to the Griffiths' transversality, we then view the differential of $\mathcal{P}$ as a map

$$
d \mathcal{P}: T_{S, s} \rightarrow \oplus_{p} \operatorname{Hom}\left(F^{p} / F^{p+1}, F^{p-1} / F^{p}\right) \subset T_{\mathcal{P}(s)} \mathbf{D} .
$$

Now $F^{p} / F^{p+1}=H^{p, q}=H^{q}\left(X_{s}, \Omega_{X_{s}}^{p}\right)$. Then, the differential of the period map is given by (see Voi07, I $\S 10.2 .3]$ ):

$$
d \mathcal{P}(\xi)(\phi)=\kappa(\psi) \cup \phi \in H^{q+1}\left(X_{s}, \Omega_{X_{s}}^{p-1}\right) \text { for } \xi \in T_{S, s} \text { and } \phi \in H^{q}\left(X_{s}, \Omega_{X_{s}}^{p}\right),
$$

where $\kappa: T_{S, s} \rightarrow H^{1}\left(X_{s}, T_{s}\right)$ is the Kodaira-Spencer map, and

$$
\cup: H^{1}\left(X_{s}, T_{s}\right) \times H^{q}\left(X_{s}, \Omega_{X_{s}}^{p}\right) \rightarrow H^{q+1}\left(X_{s}, \Omega_{X_{s}}^{p-1}\right)
$$

is induced by the contraction map $T_{s} \otimes \Omega_{X_{s}}^{p} \rightarrow \Omega_{X_{s}}^{p-1}$. As a consequence, it is not hard to prove infinitesimal Torelli in a variety of cases. An interesting case is that of Calabi-Yau threefolds. Due to the Bogomolov-Tian Theorem, the moduli space $\mathcal{M}$ of Calabi-Yau threefolds (in a fixed deformation class) is smooth and

$$
T_{s} \mathcal{M} \cong H^{1}\left(X_{s}, T_{s}\right) \cong H^{1}\left(X_{s}, \Omega_{X_{s}}^{2}\right)
$$

(using the Calabi-Yau condition: $K_{X_{s}}$ is trivial). Since $H^{3,0}\left(X_{s}\right) \cong \mathbb{C}$, it follows that $d \mathcal{P}$ gives an isomorphism between the tangent space to moduli and the subspace of horizontal directions in $T_{\mathcal{P}(s)} \mathbf{D}$. Thus, locally $\mathcal{M}$ is identified with a maximal horizontal subvariety $Z \subset \mathbf{D}\left(\right.$ N.B. $\left.\operatorname{dim} \mathcal{M}=\operatorname{dim} Z=h^{2,1}\right)$. A local description of the horizontal subvarieties $Z$ of $\mathbf{D}$ in the Calabi-Yau case was given by Bryant-Griffiths [BG83. A global description is much harder, and little is known (see however DM06] and [FL13]).

Global Torelli results ( $\mathcal{P}$ injective) are harder to obtain (as they involve the global geometry of the moduli spaces). In particular, the global Torelli is known to hold in the following cases:

- for abelian varieties (this is essentially a tautological statement);

- for curves (a classical statement), and cubic threefolds ([CG72]);

- for $K 3$ s (a nontrivial result, typically proved using the density of the Kummer surfaces in the moduli of $K 3 \mathrm{~s}$, and then applying Torelli for ppavs);

- for $K 3$ like situations: e.g. cubic fourfolds ([Voi86]) and compact Hyperkähler manifolds (due to Verbitsky, see Huy11).

It is also known that generic Torelli ( $\mathcal{P}$ is generically injective) theorem holds for most hypersurfaces (a result due to Donagi, see Voi07, II §6.3.2]). Roughly, the idea here is that the image of the period map has such a high codimension (satisfies so many infinitesimal conditions), that the hypersurfaces can be recovered from infinitesimal data. Of course, the global or even generic Torelli doesn't always hold. For example, for del Pezzos the associated VHS is trivial; there are also some non-trivial counterexamples to Torelli. In any case, it is expected that various forms of Torelli will hold quite generally. On the other hand, with few exceptions, the period map is not dominant. 
2.1.4. For moduli constructions, we restrict to the case $\mathbf{D}$ is a Hermitian symmetric domain (or more generally to the cases covered by Theorem 2.2). In conclusion, our understanding of the images of period maps outside the situation covered by Theorem 2.2 is quite limited. Consequently, the only case that we know how to use the period map for constructing a moduli space is when we have a period map

$$
\mathcal{P}: \mathcal{M} \rightarrow \mathcal{D} / \Gamma
$$

where $\mathcal{D}$ is a Hermitian symmetric domain and $\Gamma$ an arithmetic group acting on $\mathcal{D}$. Due to Baily-Borel and Borel theorems discussed below, $\mathcal{P}$ is in fact an algebraic (typically birational) map between quasi-projective varieties. While rare, this situation is particularly good due to the special structure of $\mathcal{D} / \Gamma$; some applications to moduli are explained below.

Remark 2.4. We note here that there is at least one application to using the period map in the case $\mathcal{D}$ is not Hermitian symmetric. Namely, the period domains $\mathbf{D}$ are negatively curved in the horizontal directions. Consequently, if the Torelli theorem holds, one obtains hyperbolicity results for the moduli space (see [Keb13] for a survey on hyperbolicity). Even when Torelli doesn't hold, one might obtain hyperbolicity results by using cyclic cover constructions and applying Torelli theorems on related moduli spaces (e.g. see [VZ05]).

2.2. Applications of locally symmetric varieties. The main advantage of realizing a moduli space as a locally symmetric variety $\mathcal{D} / \Gamma$ (with $\mathcal{D}$ a Hermitian symmetric domain and $\Gamma$ an arithmetic group acting on $\mathcal{D}$ ) is that such a variety has a lot of additional structure, which brings in additional tools such as the theory of automorphic forms. We briefly survey two applications of the description of moduli as locally symmetric varieties: the existence of natural compactifications and results on the Kodaira dimension of the moduli spaces.

2.2.1. Compactifications of locally symmetric varieties. A locally symmetric variety has several natural compactifications. First, any locally symmetric variety $\mathcal{D} / \Gamma$ has a canonical compactification, the Satake-Bailly-Borel compactification, which can be defined by:

$$
\mathcal{D} / \Gamma \subseteq(\mathcal{D} / \Gamma)^{*}:=\operatorname{Proj} A(\Gamma),
$$

where $A(\Gamma)$ is the ring of $\Gamma$-automorphic forms on $\mathcal{D}$ (this is a finitely generated ring by $[\mathrm{BB} 66])$. By construction $(\mathcal{D} / \Gamma)^{*}$ is a projective variety which does not depend on any choices. It is also a minimal compactification, in the sense that any normal crossing compactification of $\mathcal{D} / \Gamma$ will map to it. More precisely, the following holds (due to curvature properties of period domains):

Theorem 2.6 (Borel's Extension Theorem [Bor72]). Let $S$ be a smooth variety, and $\bar{S}$ a smooth simple normal crossing (partial) compactification of $S$. Then any locally liftable map $S \rightarrow \mathcal{D} / \Gamma$ extends to a regular map $\bar{S} \rightarrow(\mathcal{D} / \Gamma)^{*}$.

The main disadvantage of the SBB compactification $(\mathcal{D} / \Gamma)^{*}$ is that it is quite small, and thus it doesn't accurately reflects the geometry of degenerations. Also, $(\mathcal{D} / \Gamma)^{*}$ tends to be quite singular. For instance, it is well known that

$$
\mathcal{A}_{g}^{*}=\mathcal{A}_{g} \sqcup \mathcal{A}_{g-1} \cdots \sqcup \mathcal{A}_{0}
$$

and thus the boundary has codimension $g$. Similarly, for Type IV domains (i.e. $K 3$ type period domains) the boundary is 1-dimensional. 
To rectify this issue, Mumford et al. AMRT10 have introduced the toroidal compactifications $\overline{\mathcal{D} / \Gamma^{\Sigma}}$. By construction ${\overline{\mathcal{D}} / \Gamma^{\Sigma}}^{\Sigma}$ depends on a choice, an admissible rational polyhedral decomposition $\Sigma$ of a certain cone (see [Nam80, Def. 7.3]). The toroidal compactifications come equipped with natural forgetful maps

$$
\overline{\mathcal{D} / \Gamma}^{\Sigma} \rightarrow(\mathcal{D} / \Gamma)^{*}
$$

for any $\Sigma$. For suitable $\Sigma$ the compactifications $\overline{\mathcal{D} / \Gamma}^{\Sigma}$ are smooth (up to finite quotients) and projective. Of course, the main disadvantage of the toroidal compactifications $\overline{\mathcal{D}} / \Gamma^{\Sigma}$ is that there is a plethora of choices. It is unclear which of the toroidal compactifications should have a geometric meaning. The following are some known facts on the modular meaning of some toroidal compactifications:

(A) (Mumford, Namikawa [Nam80, Alexeev [Ale02]) The second Voronoi compactification $\overline{\mathcal{A}}_{g}^{\text {Vor }}$ for $\mathcal{A}_{g} \cong \mathfrak{H}_{g} / \mathrm{Sp}(2 g, \mathbb{Z})$ has a modular interpretation (in a strong functorial sense).

(C) (Mumford, Alexeev-Brunyate [AB12]) The period map $M_{g} \rightarrow \mathcal{A}_{g}$ extends to a regular $\bar{M}_{g} \rightarrow \overline{\mathcal{A}}_{g}^{\Sigma}$, where $\Sigma$ is the second Voronoi or perfect cone. It does not extend to the central cone compactification (for $g \geq 9$ ).

(P) (Friedman-Smith [FS86, see also [ABH02] and [CMGHL14]) The period map $R_{g} \rightarrow \mathcal{A}_{g}$, where $R_{g}$ is the moduli of Prym curves, does not extend to a regular map $\bar{R}_{g} \rightarrow \overline{\mathcal{A}}_{g}^{\Sigma}$ for any of the standard toroidal compactifications (here $\bar{R}_{g}$ is Beauville's admissible covers compactification of $R_{g}$ ).

For moduli of $K 3 \mathrm{~s}$, it is an open question to give geometric meaning to any of the toroidal compactifications (analogue to (A) above); the best result so far is Theorem 2.14 below. Another interesting situation is that of cubic threefolds (which are closely related to genus 4 curves and Prym varieties of genus 5). Specifically, the intermediate Jacobian for a cubic threefold is a ppav of genus 5. One is interested in understanding the closure of the intermediate Jacobian locus in some toroidal compactification $\overline{\mathcal{A}}_{5}^{\Sigma}$ (this question is related to $(\mathrm{C})$ and $(\mathrm{P})$ above); see CML09 and GH12 for some results on this topic. In particular, in CML09] we have computed the closure of the intermediate Jacobian locus in the SBB compactification $\mathcal{A}_{5}^{*}$. The key ingredients for this result are the extension Theorem 2.6 and an appropriate blow-up of the GIT compactification of the moduli space of cubic threefolds. As discussed in the following subsection, it is quite typical to use auxiliary compactifications (such as GIT) in order to understand the geometric meaning at the boundary of the period domain.

Remark 2.7. The limit of a degeneration of Hodge structures is a mixed Hodge structure $\left(H, F^{\bullet}, W_{\bullet}\right)$. From this point of view, it is known that the data encoded in the boundary of the SBB compactification $(\mathcal{D} / \Gamma)^{*}$ is equivalent to the data of the graded pieces $\mathrm{Gr}_{\bullet}^{W} H$ of the mixed Hodge structure. For instance, in a degeneration of abelian varieties the limit is a semiabelian variety (i.e. an extension $0 \rightarrow\left(\mathbb{C}^{*}\right)^{n} \rightarrow$ $X \rightarrow A \rightarrow 0$ of an abelian variety $A$ by a torus). The SBB compactification remembers only the compact part $A \in \mathcal{A}_{g-n} \subset \partial \mathcal{A}_{g}^{*}$. On the other hand, the toroidal compactification ${\overline{\mathcal{D}} / \Gamma^{\Sigma}}^{\Sigma}$ encodes the full limit mixed Hodge structure (see Cat84). This gives a conceptual explanation of why (A) is plausible. 
Remark 2.8. There exist extension theorems from normal crossing compactifications $S \rightarrow \bar{S}$ to toroidal compactifications (e.g. [Nam80, Thm. 7.29]), but in contrast to Theorem 2.6 the extension $\bar{S} \rightarrow \overline{\mathcal{D}} / \Gamma^{\Sigma}$ exists only if the cones spanned by the monodromies around the boundary divisors in $\bar{S}$ are compatible with the cones of the decomposition $\Sigma$ (see also Cat84). This is due to the fact that $\overline{\mathcal{D}} / \Gamma^{\Sigma}$ has a toric structure near the boundary.

Remark 2.9. There are two directions in which the SBB and toroidal compactifications can be generalized. First, for the Hermitian symmetric case, Looijenga [Lo003a, Loo03b] has introduced the semi-toric compactifications. Roughly, one makes a choice $\Sigma$ similar to the toroidal case, giving a compactification $\overline{\mathcal{D} / \Gamma}^{\Sigma}$, but (in contrast to the toroidal case) $\Sigma$ is only a locally rational polyhedral decomposition. The semi-toric construction generalizes both the Baily-Borel (roughly $\Sigma$ is the trivial decomposition) and the toroidal constructions ( $\Sigma$ is rational polyhedral). For an application to moduli of the semi-toric compactifications see $\$ 2.3$. In a different direction, one can ask for compactifications when $\mathcal{D}$ is not a Hermitian symmetric domain, but rather a general period domain $\mathbf{D}$. This case was analyzed by Kato-Nakayama-Usui [KU09. In this situation one doesn't get a compact space or even a variety, but a "log variety"; still this is quite useful in applications (e.g. regarding the algebraicity of various Hodge theoretic loci).

2.2.2. Using automorphic forms to obtain geometric consequences. As discussed in the previous subsection, the SBB compactification $(\mathcal{D} / \Gamma)^{*}$ is a projective variety, being the Proj of the finitely generated algebra of automorphic forms. In fact, the automorphic forms can be regarded as sections of a certain ample line bundle. If the arithmetic group $\Gamma$ acts freely (which never happens in practice), the automorphic forms would be pluricanonical forms on $\mathcal{D} / \Gamma$. Thus, it is no surprise that one can use automorphic forms to prove that various moduli spaces are of general type. In practice, since $\Gamma$ doesn't act freely on $\mathcal{D}$, one has to take care of the ramification of the natural projection $\mathcal{D} \rightarrow \mathcal{D} / \Gamma$ and of the singularities at the boundary of $(\mathcal{D} / \Gamma)^{*}$ (typically one has to pass to a toroidal compactification). The situation for ppavs is well understood. In particular,

Theorem 2.10 (Tai [Tai82, Mumford [Mum83]). $\mathcal{A}_{g}$ is of general type for $g>6$.

For small genus $(g \leq 5)$, since $\mathcal{A}_{g}$ is closely related to $M_{g}$ or $R_{g}$ and these have simple GIT models, one obtains unirationality (the case $g=6$ is open). Similar results hold also for $K 3 \mathrm{~s}$. Here, it is harder to produce automorphic forms. Essentially, all automorphic forms that occur in the study of the moduli spaces of $K 3 \mathrm{~s}$ are obtained by appropriate restrictions of the Borcherds' automorphic form $\Phi_{12}$ (see BKPSB98]). In particular, Gritsenko-Hulek-Sankaran GHS07 have proved that for $d$ large enough, $\mathcal{F}_{d}$ is of general type (see also GHS13 for a survey of related results).

The are numerous other geometric applications involving automorphic forms. For instance, we have used a restriction of the $\Phi_{12}$ form to relate Kondo's ball quotient model for $M_{4}$ to a step in the Hassett-Keel program (this is related to Theorem 1.8 and $\$ 2.3$ below). Maulik-Pandharipande [MP07] have given a generating function for the degrees of the Noether-Lefchetz loci in the moduli of K3 surfaces with applications to enumerative geometry (see also [LZ12] for the similar case of cubic 
fourfolds). Even more spectacularly, Maulik and Charles Cha12 have used related ideas to give a proof of the Tate conjecture for $K 3 \mathrm{~s}$.

In conclusion, we see that a (birational) model of type $\mathcal{D} / \Gamma$ for a moduli space has deep consequences; essentially the global results obtained by this construction are unparalleled. As already explained, the main drawback is that there is limited applicability of this construction. Another issue is that it is typical difficult to understand the geometric meaning of the compactifications $(\mathcal{D} / \Gamma)^{*}$ and $\overline{\mathcal{D}} / \Gamma^{\Sigma}$. To handle this last issue, one typically needs to construct a different (more geometric) compactification $\overline{\mathcal{M}}$ for the moduli space, and then to study the behavior of the period map at the boundary of $\overline{\mathcal{M}}$. We discuss a situation when this comparison approach works in the following subsection (see also $\$ 3.2$ ).

2.3. Comparison to GIT compactifications. As explained above, the ideal situation is to have a period map

$$
\mathcal{P}^{\circ}: \mathcal{M}^{\circ} \rightarrow \mathcal{D} / \Gamma,
$$

from a moduli space $\mathcal{M}^{\circ}$ of smooth varieties to the quotient of a Hermitian symmetric domain $\mathcal{D}$ by an arithmetic group $\Gamma$. Even after assuming that the Torelli theorem holds and that $\mathcal{P}$ is dominant (and thus $\mathcal{P}$ is a birational morphism of quasi-projective varieties) it is typically hard to understand the image of the period map and the geometric meaning of the boundary (the complement of $\operatorname{Im}\left(\mathcal{P}^{\circ}\right)$ ). We describe here a situation when we can answer these questions by using an alternative GIT compactification. As a byproduct, one obtains a dual GIT/Hodge theoretic description for a moduli space. This has the advantage of both being (weakly) geometric due to GIT, and having a lot of additional structure due to the $\mathcal{D} / \Gamma$ description.

Namely, with assumptions as above, we further restrict to the situation that $\mathcal{D}$ is either a Type IV domain (i.e. $K 3$ type) or a complex ball. What is special about these cases is that the maximal Noether-Lefschetz loci (i.e. loci where the Hodge structure is special in the sense of Mumford-Tate groups, see [GGK12, §IIC]) are Heegner divisors, i.e. codimension 1 subdomains $\mathcal{H} \subset \mathcal{D}$ of the same type as $\mathcal{D}$ (e.g. for a $n$-dimensional complex ball $\mathcal{D}$, the loci $\mathcal{H}$ will be $(n-1)$-dimensional sub-balls in $\mathcal{D}$ ). In fact, these Heegner divisors are nothing but hyperplane sections of $\mathcal{D}$ in an appropriate sense (i.e. using $\mathcal{D} \subset \check{\mathcal{D}} \subseteq \mathbb{P}^{N}$ ). In this situation $(\mathcal{P}$ birational and $\mathcal{D}$ of Type IV or ball), Looijenga Loo03b, LS07, has observed the following:

(i) $\mathcal{P}^{\circ}$ is typically an open embedding with image the complement of a $\Gamma$ invariant arrangement of hyperplanes.

(ii) Given (i), $\mathcal{P}^{\circ}$ can be (typically) extended to an explicit isomorphism between a GIT compactification of $\mathcal{M}^{\circ}$ and a semi-toric modification of $\mathcal{D} / \Gamma$.

The idea for proving these statements is to consider a GIT compactification $\overline{\mathcal{M}}$ of $\mathcal{M}^{\circ}$ which almost agrees with $(\mathcal{D} / \Gamma)^{*}$ as a polarized variety. More specifically, typically the period map $\mathcal{M}^{\circ} \rightarrow \mathcal{D} / \Gamma$ extends naturally to a larger moduli stack $\mathcal{M}$ of very mildly singular varieties. For example, for polarized $K 3$ surfaces the period map extends naturally to the moduli $\mathcal{M}$ of polarized $K 3$ s with ADE singularities. Then one constructs a GIT compactification $\overline{\mathcal{M}}$ such that $\mathcal{M} \subset \overline{\mathcal{M}}$ has boundary of codimension 2 and with the right polarization (explained below). The GIT quotients typically satisfy the first condition, as their boundary is quite small (however this is typically not true for the smooth locus $\mathcal{M}^{\circ} \subset \overline{\mathcal{M}}$ ). Then one considers the 
birational map

$$
\mathcal{P}: \overline{\mathcal{M}} \rightarrow(\mathcal{D} / \Gamma)^{*},
$$

which is regular on $\mathcal{M}$. Using the GIT semistable replacement lemma 1.7, one proves (i) or even

$$
\mathcal{M} \cong(\mathcal{D} \backslash \mathcal{H}) / \Gamma
$$

as quasi-projective varieties. Roughly, one shows that the limit Hodge structure for a geometric degeneration with central fiber in a list (determined by GIT) will be either not pure (and thus will go to the boundary of $\mathcal{D} / \Gamma$ ) or it will be Hodge special, and thus it will belong to a Noether-Lefschetz locus (a hyperplane in $\mathcal{D}$ ).

Having the right polarization for the GIT quotient $\overline{\mathcal{M}}$ means that the isomorphism 2.11 preserves the line bundles induced from the projective varieties $\overline{\mathcal{M}}$ and $(\mathcal{D} / \Gamma)^{*}$ respectively. By construction, we have that $\mathcal{M} \subset \overline{\mathcal{M}}$ has boundary of codimension at least 2. If the same were true for $(\mathcal{D} \backslash \mathcal{H}) / \Gamma \subset(\mathcal{D} / \Gamma)^{*}$ (e.g. if $\mathcal{H}=\emptyset)$, then due to the extension of sections of line bundles in codimension 2 , we would get $\overline{\mathcal{M}} \cong(\mathcal{D} / \Gamma)^{*}$. In general the hyperplane arrangement $\mathcal{H}$ is nontrivial and thus $\mathcal{H} / \Gamma$ is a divisor. Looijenga noted that by using an appropriate semi-toric compactification $\overline{\mathcal{D} / \Gamma}^{\Sigma}$ (where the decomposition $\Sigma$ is induced by the arrangement $\mathcal{H}$ ), one obtains that the divisor $\mathcal{H} / \Gamma$ is $\mathbb{Q}$-Cartier and negative (and thus can be contracted). By this process, Looijenga obtains a modification $\overline{(\mathcal{D} / \Gamma)}_{\mathcal{H}}$ of $(\mathcal{D} / \Gamma)^{*}$ with the property that it preserves $(\mathcal{D} \backslash \mathcal{H}) / \Gamma$, but contracts $\mathcal{H} / \Gamma$ to a smaller dimensional stratum (and thus the codimension 2 argument holds). We conclude

$$
\overline{\mathcal{M}} \cong{\overline{(\mathcal{D} / \Gamma)_{\mathcal{H}}}}
$$

(see [Loo03b, Thm. 7.6] for a precise statement and precise assumptions).

Remark 2.13. Similarly to the definition 2.5 for the $\mathrm{SBB}$ compactification $(\mathcal{D} / \Gamma)^{*}$, one can describe $\overline{(\mathcal{D} / \Gamma)_{\mathcal{H}}}$ as the Proj of a ring of meromorphic automorphic forms with poles along $\mathcal{H}$. More explicitly, $\overline{(\mathcal{D} / \Gamma)_{\mathcal{H}}}$ is obtained by $(1)$ doing a small partial resolution of the boundary $(\mathcal{D} / \Gamma)^{*}$ so that $\mathcal{H} / \Gamma$ becomes $\mathbb{Q}$-Cartier, followed by $(2)$ blowing up the arrangement $\mathcal{H}$ to normal crossing and finally (3) contracting in the opposite direction (i.e. the arrangement is "flipped": linear strata in the arrangement $\mathcal{H}$ are replaced in a way that changes the dimension with codimension). The main points are that $\overline{(\mathcal{D} / \Gamma)}_{\mathcal{H}}$ is easy to describe in practice (e.g. see Theorem 2.14 below) and that this space has a structure (e.g. various arithmetic stratifications) similar to $(\mathcal{D} / \Gamma)^{*}$.

The simplest example of 2.12 is the moduli space of elliptic curves. On one hand, we can give a compactification $\overline{\mathcal{M}}$ for this moduli space by using the GIT quotient for plane cubics. On the other hand, the moduli space of elliptic curves can be described as the quotient $\mathfrak{h} / \mathrm{SL}(2, \mathbb{Z})$ of the Siegel upper half space by the modular group. As it is well known, we have

$$
\bar{M}_{1} \cong \mathbb{P}^{1} \cong(\mathfrak{H} / \mathrm{SL}(2, \mathbb{Z}))^{*} \cong \mathbb{P} \operatorname{Sym}^{3} V^{*} / / \operatorname{SL}(3, \mathbb{C}) .
$$

Some analogue results, when a GIT quotient $\overline{\mathcal{M}}$ is isomorphic to a Baily-Borel compactification $(\mathcal{D} / \Gamma)^{*}$ (i.e. the arrangement $\mathcal{H}=\emptyset$ in $(2.12)$ ) hold for some of the $M_{0, n}$ with $n \leq 12$ (Deligne-Mostow [DM86]) and the moduli of cubic surfaces (cf. [ACT02, DvGK05]). In general, the hyperplane arrangement $\mathcal{H}$ is non-trivial, and thus the GIT quotient $\overline{\mathcal{M}}$ is isomorphic to a semi-toric modification of $\mathcal{D} / \Gamma$ 
(which is a non-trivial explicit modification of $\left.(\mathcal{D} / \Gamma)^{*}\right)$. Specifically, the following moduli spaces are known to have a dual GIT/Hodge theoretic description:

- $M_{g}$ for $g \leq 4$ and $g=6$ (Kon00, Loo07, Kon02, CMJL12a, AK11]);

- moduli of del Pezzo surfaces ( ACT02, DvGK05, [HL02);

- moduli of low degree $K 3$ surfaces ([Sha80, Sha81a], Loo03b, §8.2]);

- moduli of cubic threefolds ([All03, ACT11, [LS07]);

- moduli of cubic fourfolds (Laz09, Laz10, [Loo09]).

The example of degree $2 K 3$ surfaces is discussed in some detail below.

We note that the comparison result 2.12) is quite surprising given the different nature of the objects under consideration: on one hand the GIT quotient is purely algebraic, while $\mathcal{D} / \Gamma$ is of analytic and arithmetic nature. This explains to a certain extent the relative scarcity of such examples.

2.3.1. An explicit example - the moduli of degree $2 \mathrm{~K} 3$ surfaces. Let $\mathcal{F}_{2}$ be the moduli space of degree $2 K 3$ surfaces. As it is well known, the period map gives an isomorphism (of quasi-projective varieties):

$$
\mathcal{F}_{2} \cong \mathcal{D} / \Gamma
$$

where $\mathcal{D}$ is a 19-dimensional Type IV domain, and $\Gamma$ an arithmetic group. As discussed in $\$ 2.2 .1, \mathcal{D} / \Gamma$ has several compactifications, but neither of them is known to be geometric. Shah Sha80 has constructed a different model (compact and with weak geometric meaning) for $\mathcal{F}_{2}$ by using GIT. Specifically, a degree $2 K 3$ surface is a double cover of $\mathbb{P}^{2}$ branched along a plane sextic. Thus, the GIT quotient $\overline{\mathcal{M}}$ for plane sextics is a birational model for $\mathcal{F}_{2}$. Not all degree $2 K 3$ surfaces are double covers of $\mathbb{P}^{2}$; the so called unigonal $K 3$ s (which form a divisor in $\mathcal{F}_{2}$ ) correspond in $\overline{\mathcal{M}}$ to the triple conic. More precisely, the triple conic is semistable with closed orbit; we denote by $\omega \in \overline{\mathcal{M}}$ the corresponding point in the GIT quotient. Shah has shown that the exceptional divisor of a blow-up $\widehat{\mathcal{M}}$ of $\omega \in \overline{\mathcal{M}}$ (in modern language, the Kirwan blow-up of $\omega$ ) corresponds to the unigonal degree $2 \mathrm{~K} 3 \mathrm{~s}$. It follows that the GIT space $\widehat{\mathcal{M}}$ is a compactification of $\mathcal{F}_{2}$ with explicitly described boundary.

From the Hodge theoretic perspective, $\mathcal{F}_{2} \cong \mathcal{D} / \Gamma$ has a canonical compactification, the SBB compactification $(\mathcal{D} / \Gamma)^{*}$. In this situation the boundary of $(\mathcal{D} / \Gamma)^{*}$ consists of 4 modular curves meeting in a point. On the other hand, the boundary of $\mathcal{F}_{2}$ in the GIT compactification $\widehat{\mathcal{M}}$ also has 4 boundary components, but some of dimension 1 and some of dimension 2 (see figure 1 ). In other words, $\widehat{\mathcal{M}}$ and $(\mathcal{D} / \Gamma)^{*}$ almost agree everywhere. The explanation for this fact is given by the general framework of Looijenga discussed above. Specifically, the following holds:

Theorem 2.14 (Looijenga, Shah). The open embeddings $\mathcal{F}_{2} \subset \widehat{\mathcal{M}}$ and $\mathcal{F}_{2} \subset$ $(\mathcal{D} / \Gamma)^{*}$ extend to a diagram (with regular maps):

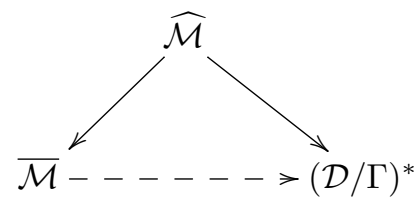

such that

i) $\widehat{\mathcal{M}} \rightarrow \overline{\mathcal{M}}$ is the partial Kirwan blow-up of $\omega \in \overline{\mathcal{M}}$; 


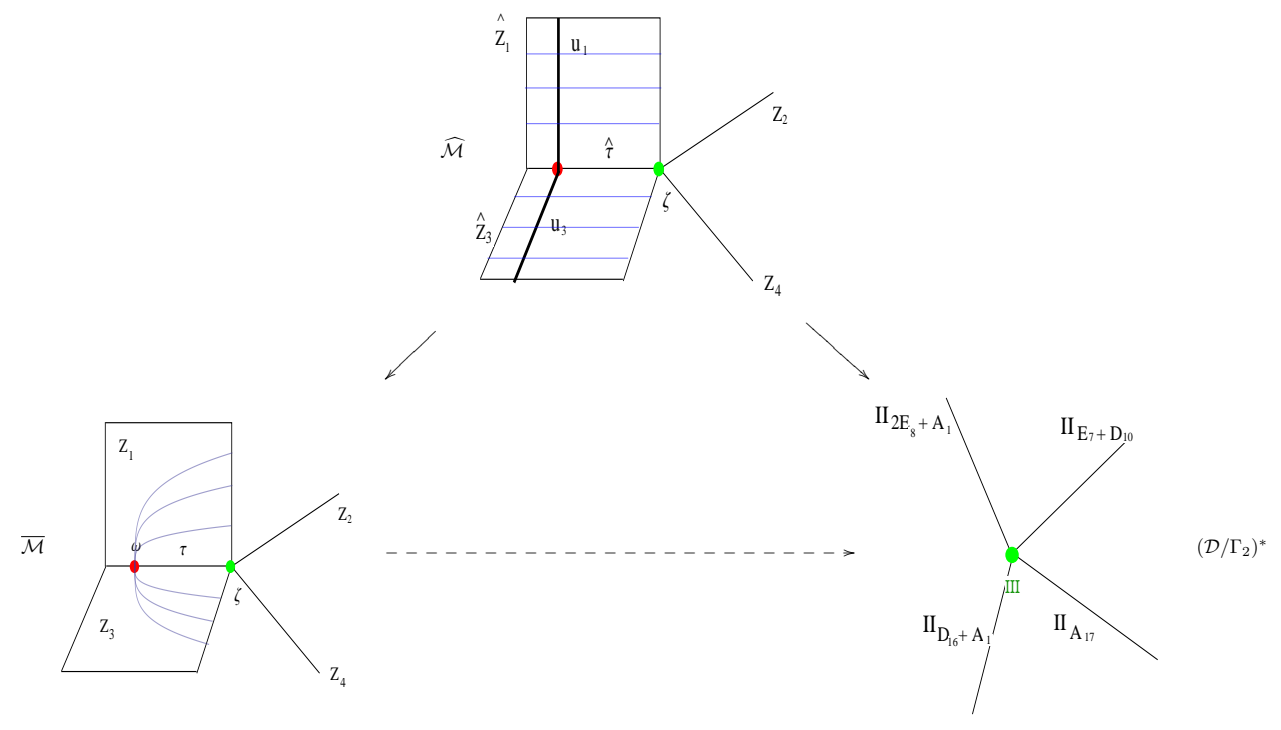

Figure 1. The boundary strata in the GIT and Baily-Borel compactifications for degree $2 K 3$ surfaces

ii) $\widehat{\mathcal{M}} \rightarrow(\mathcal{D} / \Gamma)^{*}$ is the Looijenga modification of the Baily-Borel compactification associated to the hyperplane arrangement $\mathcal{H}_{\infty}$ which corresponds to the divisor of unigonal K3s; more intrinsically, it is a small modification of $(\mathcal{D} / \Gamma)^{*}$ such that the closure of Heegner divisor $\mathcal{H}_{\infty} / \Gamma$ becomes $\mathbb{Q}$-Cartier.

iii) The exceptional divisor of $\widehat{\mathcal{M}} \rightarrow \overline{\mathcal{M}}$ maps to the unigonal divisor.

iv) The boundary components are mapped as in Figure 1.

Remark 2.15. We note that the birational map $\overline{\mathcal{M}} \rightarrow(\mathcal{D} / \Gamma)^{*}$ is everywhere defined except the point $\omega$. After the Kirwan blow-up $\widehat{\mathcal{M}} \rightarrow \overline{\mathcal{M}}$ of $\omega$, one gets a regular map. The explanation for this fact is that, except for the triple conic, all the degenerations occurring in the GIT quotient $\overline{\mathcal{M}}$ are "mild". This was first observed by Shah Sha79, Sha80. In modern language, this is related to the theory of du Bois singularities (see 3.2 ).

\section{The KSBA Approach to Moduli Spaces}

The previous two approaches to constructing moduli spaces are global in nature. Essentially, these approaches give a quasi-projective variety $\mathcal{M}$ parameterizing the generic objects of a moduli problem, and a compactification $\overline{\mathcal{M}}$ with little control on the modular meaning of the boundary. The KSBA approach reviewed below takes the opposite point of view. It starts by identifying the correct boundary objects, and then it constructs a moduli space by gluing the local deformations of "stable" objects in a stack. It is of significant interest to reconcile this approach with the other two approaches (thus gaining modularity at the boundary, as well as nice global properties). This topic is still in early stages of development. We briefly discuss below the du Bois singularities which link to the Hodge theoretic approach, and K-stability which links to GIT. 
The original KSBA approach was introduced in Kollár-Shepherd-Barron KSB88. and Alexeev [Ale96]. A recent survey of the KSBA approach is Kollár Kol13a]. A two volume monograph (see Kol13b) handling the more delicate aspects of the KSBA approach will appear soon. A detailed example (including a discussion of the state-of-art theoretical background) is discussed in Aleexev's lectures Ale13.

3.1. The KSBA approach. The KSBA approach is a natural generalization of the Deligne-Mumford construction DM69 of the proper stack of stable curves $\overline{\mathcal{M}}_{g}$. We review this approach below focusing on the main ideas of the construction and ignoring various technical issues. We caution the reader that some of these technical are quite subtle in higher dimensions and should consult Kol13a, Kol13b for precise statements.

3.1.1. The original construction of $\overline{\mathcal{M}}_{g}$ does not involve GIT.. The abstract point of view to moduli is to define a moduli functor, i.e. to define a class of objects of interest and allowable families of objects (e.g. flat proper morphisms $\mathcal{X} / S$ ). Under mild hypotheses, there is an associated algebraic moduli stack $\mathcal{M}$, which is roughly obtained by gluing the (allowable) deformation spaces (= local patches of $\mathcal{M}$ ) of the objects under consideration. From this point of view, the main issue is to select a class of objects so that the moduli stack is proper and separated. This is a delicate balance, as it is easy to achieve either of those two conditions, e.g. smooth varieties of general type (with ample $K_{X}$ ) vs. GIT semistable varieties (say with respect to the embedding given by $n K_{X}$ ), but not both at the same time. By the valuative criteria, defining a proper and separated moduli stack is essentially equivalent to asking that a 1-parameter family $\mathcal{X}^{*} / \Delta^{*}$ over the punctured disk has a unique limit with respect to the given moduli functor.

In the case of curves, it is well known that the correct class of objects to define a proper moduli stack $\overline{\mathcal{M}}_{g}$ is that of stable curves $C$ :

(S) $C$ is a curve with only nodal singularities;

(A) every rational component contains at least 3 special points, or equivalently $|\operatorname{Aut}(C)|<\infty$, or equivalently $\omega_{C}$ is ample.

This follows from considering the following three steps:

Step 0: Semi-stable reduction - after a finite base change we can assume $\mathcal{C} / \Delta$ is semistable family (i.e. $\mathcal{C}$ is smooth with reduced normal crossing central fiber). This is a nice topological and geometric model, which is the starting point for the next steps. Of course, the issue here is that $\mathcal{C}$ is far from unique (e.g. due to blow-ups).

Step 1: Relative minimal model $-\mathcal{C}$ is a surface, and thus the minimal model can be easily obtained by contracting the $(-1)$-curves in the fibers. Here the total space $\mathcal{C}$ is still smooth, but the moduli functor is not yet separated.

Step 2: Relative canonical model - this is obtained by contracting the (-2)-curves orthogonal to $\omega_{\mathcal{C} / \Delta}$. Here, we obtained separateness, but we need to allow mild singularities (rational double points) for the total space.

Remark 3.1. The main facts leading to nodal curves as the correct class of degeneration for curves are: (a) the relative canonical model agrees with the log canonical model of the pair $\left(C, C_{0}\right)$, and then (b) by adjunction, it follows that $C_{0}$ has semi-log canonical singularities and hence $C_{0}$ is nodal. 
In general, Step 0 is true in high generality (cf. AMRT10). Steps 1 and 2 are then achieved (under relatively mild assumptions) by using the full strength of the Minimal Model Program (MMP; [BCHM10]). We emphasize that for Step 2 it is essential to have varieties of general type (or log general type). For instance, we note that for $K 3$ surfaces there exists a very good answer to steps 0 and 1 :

Theorem 3.2 (Kulikov-Persson-Pinkham/Shepherd-Barron FM83]). Let $(\mathcal{X}, \mathcal{L}) / S$ be a 1-parameter degeneration of polarized $K 3$ surfaces. After a finite base change and birational transformations, one can assume the following:

i) $\mathcal{X}$ is a semistable family;

ii) $K_{\mathcal{X} / B} \equiv 0$;

iii) $\mathcal{L}$ is nef.

However, there is no good analogue to Step 2. Namely, one can define $\overline{\mathcal{X}}=$ $\operatorname{Proj} R\left(\pi_{*} \mathcal{L}\right)$, which is quite similar to a relative log canonical model, but even so one does not obtain a separated moduli stack. If one considers instead of the line bundle $\mathcal{L}$ a divisor $\mathcal{H}$ (with $\mathcal{L}=\mathcal{O}_{\mathcal{X}}(\mathcal{H})$ ) the moduli problem becomes a problem about surfaces of log general type and it has a good solution (see Laz12]).

3.1.2. Due to MMP, the Deligne-Mumford approach "easily" generalizes in higher dimensions. As discussed above, for varieties of (log) general type the Steps 0-2 from the construction of $\overline{\mathcal{M}}_{g}$ go through also in higher dimensions. Analogous to case of curves, the correct limits of smooth varieties of general type are the KSBA stable varieties $X$ that satisfy two conditions analogue to the stable curve conditions:

(S) $X$ has semi-log-canonical singularities (or more precisely $X$ is an slc variety, see Kol13a, Def. 3.1]);

(A) $\omega_{X}$ is ample.

Note that the similarity to curves is somewhat deceiving. There are several subtle differences that make the results in higher dimension to be much harder than those for curves. For instance, even to define the conditions (S) and (A), one needs to assume: $K_{X}$ is $\mathbb{Q}$-Cartier (N.B. the nodal curves are Gorenstein; so the issue does not arise). Similarly, while all stable curves are smoothable, this is not true for stable varieties in higher dimensions. This leads to various issues: If one restricts to the smoothable stable varieties, there is no natural scheme structure at the boundary. On the other hand, if one considers all KSBA stable varieties, the application of MMP as sketched above can fail on the components of the moduli that parameterized non-smoothable slc varieties. A different method to handle these components is needed (see Kol13a, §5.2]).

Another set of issues in higher dimensions is the correct definition of the moduli functor (i.e. admissible families). While for curves, the natural requirement of flat and proper morphism suffices, in higher dimensions one has to impose additional conditions (such as the total space of a family has to be $\mathbb{Q}$-Gorenstein). We refer to Kol13a, (4.3),(4.4)] for a discussion of the moduli functor. Some of the more subtle issues were only recently settled. We refer the interested reader to Kol13a] and the upcoming companion monograph.

The upshot of this discussion is that, due to deep results in birational geometry and substantial additional work, there exists a proper and separated moduli stack $\mathcal{M}_{h}$ for varieties of $(\log )$ general type (with Hilbert polynomial $h$ ) which generalizes 
$\overline{\mathcal{M}}_{g}$. Also, since a KSBA stable variety has no infinitesimal automorphisms (e.g. BHPS13, Lemma 2.5]), $\mathcal{M}_{h}$ is a Deligne-Mumford stack. Consequently $\mathcal{M}_{h}$ has associated an algebraic space as coarse moduli space ([KM97]). Unfortunately, as discussed below the analogy to $\overline{\mathcal{M}}_{g}$ stops here.

3.1.3. The only property of $\bar{M}_{g}$ that generalizes in higher dimensions is the projectivity. The moduli stack $\overline{\mathcal{M}}_{g}$ is a proper smooth Deligne-Mumford stack, with a coarse projective moduli stack $\bar{M}_{g}$ (which, as previously discussed, can be constructed via GIT). Additionally, the boundary of $\overline{\mathcal{M}}_{g}$ is a divisor with normal crossings. Unfortunately, no general smoothness result can hold for the moduli of varieties of general type. The reason for this is that the deformation spaces even for smooth varieties can behaved arbitrarily badly (i.e. on the versal deformation spaces one can encounter essentially any singularity that can be defined over $\mathbb{Z}$ ). This is the content of Vakil's results:

Theorem 3.3 (Vakil Vak06). The following moduli spaces satisfy Murphy's law.

M2a. the versal deformation spaces of smooth $n$-folds (with very ample canonical bundle), $n \geq 2$.

M3. the Hilbert scheme of nonsingular surfaces in $\mathbb{P}^{5}$, and the Hilbert scheme of surfaces in $\mathbb{P}^{4}$.

The first result says that arbitrarily bad singularities can occur even for moduli of smooth surfaces of general type. The second result is relevant for the GIT constructions. Of course, as previously discussed, the GIT compactifications will tend to have even worst singularities than the KSBA compactifications.

One deep positive result is Kollár projectivity [Kol90 of the coarse moduli space (see also [Fuj12]):

Theorem 3.4 (Kollár, Fujino). The moduli functor of stable $\mathcal{M}_{h}$ varieties with Hilbert function $h$ is coarsely represented by a projective algebraic scheme.

Remark 3.5. The methods of Kollár use essentially that $\mathcal{M}_{h}$ is proper. It is not possible to prove (by those methods) the quasi-projectivity of the smooth locus without first compactifying the moduli space. On the other hand, as already noted, Gieker Gie77a proved (using GIT) the quasi-projectivity of the moduli space of smooth surfaces of general type. A similar higher-dimensional result (but via significantly different GIT methods) was proved by Viehweg (Vie95]). However, even in these quasi-projectivity results, there is a compactification (i.e. a GIT compactification) in the picture. In other words, we see here a manifestation of the well known principle in algebraic geometry: even if we only care about the smooth locus, it is important to have a compactification.

3.1.4. It is quite difficult to understand the KSBA compactification. In fact, few concrete examples are known. The GIT approach might help. The bad behavior of the moduli of varieties of general type discussed in the previous section has to do more with the pathology of the deformation spaces rather than the moduli itself. One might hope that for nice classes of varieties there will be a good moduli space with applications similar to those of $\bar{M}_{g}$. Unfortunately, very few examples of KSBA compacifications for moduli spaces are known. Namely, while a number of interesting illustrations of the KSBA compactification procedure were given (e.g. Hac04, vO05, [HKT09, AP09]), all these examples tend to be special in the 
sense that they are related to curves or hyperplane arrangements, etc. It is of interest to give more "generic" examples (such as moduli of quintic surfaces, or other surfaces with small $p_{g}$ and $q=0$ ) of compactifications for moduli of surfaces of general type.

The KSBA compactification is clearly the "correct" compactification for varieties of general type. However, its abstract definition makes it somewhat intractable. (What is special for $\overline{\mathcal{M}}_{g}$ is that a stable genus $g$ is obtained from lower genus curves via a simple combinatorial gadget, the dual graph, and then one can proceed inductively. For surfaces and higher dimensions, the situation is exponentially more involved.) Part of the message of these notes is to advocate that it might be possible to arrive at a KSBA compactification via interpolation. For instance, for quintic surfaces, a GIT compactification is readily available. In general, for GIT the numerical criterion gives an algorithmic way of determining the semistable points. No algorithmic procedure is known for KSBA. Thus, GIT tends to be more accessible. To interpolate from the GIT to the KSBA compactification, one can apply the replacement lemma 1.7 and reduce to the study of 1-parameter families $\mathcal{X} / \Delta$ with semistable (even with closed orbit) central fiber $X_{0}$ but not log canonical. The question then is to find the KSBA replacement for $X_{0}$ (for related work for curves see Has00).

Returning to the example of quintic surfaces, Patricio Gallardo Gal13] and Julie Rana have shown that the KSBA replacement for quintic surfaces $X_{0}$ with a unique Dolgachev singularity (those are GIT stable) are of type $\widetilde{X}_{0} \cup S$, where $\widetilde{X}_{0}$ is the resolution of $X_{0}$ and $S$ is a $K 3$ surface ( $S$ is a " $K 3$ tail", which depends on the degeneration). These type of surfaces give divisors in the KSBA compactification for quintics. It is interesting to note that these type of example were encountered also in work of Friedman [Fri83 and Shepherd-Barron who were concerned with the Hodge theoretic properties of degenerations of quintics. One wonders if the period map approach to moduli would not shed further light on the compactification problem for quintics by bringing in tools such as representation theory.

Remark 3.6. A simple connection between GIT and KSBA was observed by Hacking Hac04 and Kim-Lee [KL04]. Namely, for hypersurfaces $V \subset \mathbb{P}^{n}$ of degree $d$ : if $\left(\mathbb{P}^{n}, \frac{n+1}{d} V\right)$ is a log canonical pair, then $V$ is GIT semistable. Similarly, we have if $\left(\mathbb{P}^{n},\left(\frac{n+1}{d}+\epsilon\right) V\right)$ (for $\left.0<\epsilon \ll 1\right)$ is a log canonical pair, then $V$ is GIT stable. The converse is not true, an example being given by the triple conic viewed as a plane sextic. The reason for this is that the log canonical threshold and the numerical function $\mu(x, \lambda)$ are computed in the same way by minimizing a certain quantity over all choices of coordinates. The difference is that for GIT only linear changes of coordinates are allowed vs. analytic changes of coordinates in the KSBA case. In conclusion, GIT allows worst singularities (which are not detected by linear coordinates) for the objects in the boundary and consequently more collapsing (resulting in loss of geometric information on the degenerations).

3.2. Slc singularities are du Bois (connection to Hodge theory). From a topological/Hodge theoretic point of view, the good models for studying degenerations are the semistable models $\mathcal{X} / \Delta$. In this situation, the Clemens-Schmid exact sequence (e.g. Mor84]):

$$
\cdots \rightarrow H^{n}\left(X_{0}\right) \rightarrow H_{\lim }^{n} \stackrel{N}{\rightarrow} H_{\lim }^{n} \rightarrow H_{n}\left(X_{0}\right) \rightarrow \ldots
$$


(where $N=\log T$ is the monodromy, and $H_{\lim }^{n}$ is the limiting mixed Hodge structure) says that the limiting Hodge structure is essentially determined by the mixed Hodge structure of the central fiber $X_{0}$. The KSBA limits are obtained from $\mathcal{X} / \Delta$ by considering the relative $\log$ canonical model $\mathcal{X}^{c} / \Delta$. It is natural to ask what is the connection between the KSBA limits and the Hodge theoretic limits. In particular, it is interesting to understand the connection between the MHS on $X_{0}^{c}$ and $H_{\mathrm{lim}}^{n}$. If these questions have satisfactory answers, then, in principle, by using the structure on the Hodge theoretic side (see Section 2) one gets information of the KSBA compactification. Unfortunately, since $X_{0}^{c}$ and $\mathcal{X}^{c}$ are quite singular, the situation is not completely understood. We described below a step towards a comparison between KSBA and Hodge limits, but much remains to be done.

Since the KSBA stable curves are nodal and $\overline{\mathcal{M}}_{g}$ is a smooth normal crossing compactification, the KSBA and Hodge theoretic models are closely related in dimension 1. Namely, as discussed in $\$ 2.2 .1$, there is an extended Torelli morphism

$$
\overline{\mathcal{P}}: \bar{M}_{g} \rightarrow \overline{\mathcal{A}}_{g}^{\text {Vor }}
$$

which corresponds to class $\lambda$ on $\bar{M}_{g}$ (and thus $\lambda$ is semi-ample). For surfaces, Mumford and Shah Sha79] noted that a certain class of singularities (i.e. Gorenstein slc singularities in modern language) are cohomologically insignificant singularities. Steenbrink Ste81] then interpreted this statement as saying that (in dimension 2) the Gorenstein slc singularities are $d u$ Bois singularities. The du Bois singularities are a class of singularities that behave well from a Hodge theoretic point of view. Roughly speaking, if the central fiber $X_{0}^{c}$ has du Bois singularities, then the natural comparison map

$$
\mathrm{Gr}_{n}^{W} \operatorname{sp}_{n}: \operatorname{Gr}_{n}^{W} H^{n}\left(X_{0}^{c}\right) \rightarrow \operatorname{Gr}_{n}^{W} H_{\lim }^{n}
$$

is an isomorphism. Shah [Sha80] has used this fact to study the moduli of degree $2 K 3$ surfaces (see the discussion of $\$ 2.3$.

It turns out that the results for surfaces (slc $\Longrightarrow$ du Bois) generalize well. Specifically, Kollár and Kovács have obtained the following general result:

Theorem 3.9 (Kollár-Kovács [KK10, Thm. 1.4], Kol13b, Cor. 6.32]). Let $(X, \Delta)$ be an slc pair. Then $X$ is du Bois.

As noted, one application of the previous theorem is to understand images of period maps (see 82.3 ). Conversely, using Hodge theory, one obtains results on the KSBA moduli. For instance, the following results, which settles several technical issues in the KSBA construction, is obtained by using du Bois singularities.

Theorem 3.10 (Kollár, Kovács, cf. [Kol13b, p. 263]). Let $f: \mathcal{X} \rightarrow S$ be a proper and flat morphism with slc fibers over closed points; $S$ connected.

(1) Let $\mathcal{L}$ an $f$-semi-ample line bundle on $\mathcal{X}$ (e.g. $\mathcal{L}=\mathcal{O}_{\mathcal{X}}$ ). Then, for all $i$,

(a) $R^{i} f_{*}\left(\mathcal{L}^{-1}\right)$ is locally free and compatible with base change and

(b) $h^{i}\left(X_{s}, L_{s}^{-1}\right)$ is independent of $s \in S$.

(2) If one fiber of $f$ is Cohen-Macaulay then all fibers of $f$ are Cohen-Macaulay.

(3) $\omega_{\mathcal{X} / S}$ exists and is compatible with base change. Furthermore, for all $i$,

(a) $R^{i} f_{*} \omega_{\mathcal{X} / S}$ is locally free and compatible with base change and

(b) $h^{i}\left(X_{s}, \omega_{X_{s}}\right)$ is independent of $s \in S$. 
3.3. Asymptotic stability, K-stability, and KSBA. We close our survey with a brief discussion of the connection between (appropriate) GIT stability and KSBA stability. For further details and a more technical discussion, we recommend the survey Oda12].

3.3.1. Asymptotic stability and KSBA. The following theorem can be regarded as a comparison theorem between the GIT and KSBA approach. It says that if a canonically polarized variety $X_{0}$ is asymptotically semistable (even in a weak sense) then it is also KSBA stable.

Theorem 3.11 (Wang-Xu WX12). Let $\mathcal{X} / S$ be a KSBA-stable family over a smooth curve $S$, and $o \in S$ a special point. Assume that the fibers $X_{s}$ for $s \in S^{\circ}=$ $S \backslash\{o\}$ are asymptotically (Chow) stable. Let $r$ be such that $r K_{\mathcal{X}}$ is Cartier. Then for any flat family $\left(\mathcal{X}^{\prime}, \mathcal{L}\right) / S$ of polarized varieties which agrees with $\left(\mathcal{X}, \omega_{\mathcal{X}}^{[r]}\right)$ over $S^{\circ}$ and which has the property that the special fiber $\left(X_{o}, L_{o}^{k}\right)$ is Chow semistable for infinitely many $k>0$, we have $\left(\mathcal{X}^{\prime}, \mathcal{L}\right) \cong\left(\mathcal{X}, \omega_{\mathcal{X}}^{[r]}\right)$ (i.e. agrees everywhere).

Unfortunately, the converse can not hold. It was observed by Mumford (Mum77, Prop. 3.4], [Sha81b, Prop. 3.1]) that

Proposition 3.12 (Mumford). An asymptotically semistable surface has singularities of multiplicity at most 6.

At the same time, there are KSBA stable surfaces with singularities of higher multiplicity. Together with the theorem above, this implies a negative result:

Corollary 3.13. Assume $X_{0}$ is a KSBA stable surface which is not asymptotically semistable. Then $X_{0}$ has no asymptotic semistable replacement.

In other words, the asymptotic GIT quotients will not stabilize (as the embedding gets higher and higher); for some concrete examples of this see WX12] (also VZ10).

Remark 3.14. In general, it is very hard to analyze the asymptotic stability. The best positive result so far is a hard result of Gieseker which says that a smooth surface of general type is asymptotically stable. More precisely, the following holds: (Gieseker [Gie77a]) Let $X$ be a surface of general type. For any sufficiently large n, there is an $m$ so that the $m^{\text {th }}$ Hilbert point of the $n$ canonical image of $X$ is stable. Furthermore, $m$ and $n$ depend only on $K^{2}$ and $\chi\left(\mathcal{O}_{X}\right)$.

3.3.2. K-Stability. In recent years, motivated by the work of Donaldson, Tian, and Yau on the existence of special metrics, a new notion of stability, K-stability, has emerged (e.g. Don02). Namely, conjecturally, a polarized Kähler manifold $(X, L)$ admits a constant scalar curvature metric with class $c_{1}(L)$ iff $(X, L)$ is $K$-polystable. This is an area of very active research with many important recent results, but this goes beyond the purpose of this survey. We only touch here on the connection between K-stability and algebraic geometry. This is mostly based on work of Odaka (see Oda12 for a related survey).

The K-stability can be viewed as a refined notion of asymptotic stability. In fact, the set-up for K-stability is quite similar to that of the numerical criterion from GIT. Namely, one considers test configurations, i.e. 1-parameter families $\mathcal{X} / \mathbb{A}^{1}$ equivariant with respect to a $\mathbb{C}^{*}$ action (with $\mathbb{C}^{*}$ acting in the standard way on $\mathbb{A}^{1}$, and $X_{t} \cong X_{t^{\prime}}$ for $t, t^{\prime} \neq 0$ ). To such a test configuration, one associates a 
numerical invariant, the Donaldson-Futaki invariant $D F(\mathcal{X})$ (analogue to $\mu(x, \lambda)$ ). Then, as in the GIT numerical criterion 1.4 the $\mathrm{K}$-(semi)stablity is equivalent to the positivity/non-negativity of the Donaldson-Futaki invariant for all non-trivial test configurations.

It turns out that K-stability is essentially equivalent to KSBA stability. Specifically, the following hold:

Theorem 3.15 (Odaka Oda13, Thm. 1.2]). Let $X$ be a projective scheme satisfying $\left(^{*}\right)$ (see Oda13, Def. 1.1]) and $L$ be an ample line bundle on $X$. Then, if $(X, L)$ is $K$-semistable, $X$ has slc singularities.

(The condition $\left(^{*}\right)$ encodes the pre-conditions on $X$ to even make sense to say that $X$ has slc singularities.)

Theorem 3.16 (Odaka Oda13, Thm. 1.5]). The following hold:

i) A slc polarized variety $(X, L)$ with numerically trivial canonical divisor $K_{X}$ is K-semistable.

ii) A slc canonically polarized variety $\left(X, K_{X}\right)$ is $K$-stable.

The main drawback of K-stability is that it is not known how it can be used to construct a moduli space. For instance, it is not known if it is an open condition. Regardless, it can be viewed as an interpolation between two algebro-geometric stability conditions: GIT and KSBA stability. One wonders if it might be possible to set-up a slight modification of the asymptotic stability to obtain K-stability (and thus KSBA stability). An example (in spirit) going in this direction is OSS12, but much remains to be done.

\section{REFERENCES}

[AB12] V. Alexeev and A. Brunyate, Extending the Torelli map to toroidal compactifications of Siegel space, Invent. Math. 188 (2012), no. 1, 175-196.

[ABH02] V. Alexeev, Ch. Birkenhake, and K. Hulek, Degenerations of Prym varieties, J. Reine Angew. Math. 553 (2002), 73-116.

$\left[\mathrm{ACG}^{+} 13\right]$ D. Abramovich, Q. Chen, D. Gillam, Y. Huang, M. Olsson, M. Satriano, and S. Sun, Logarithmic Geometry and Moduli, Handbook of Moduli I, Advanced Lectures in Mathematics, vol. 24, Int. Press, Somerville, MA, 2013, pp. 1-61.

[ACT02] D. Allcock, J. A. Carlson, and D. Toledo, The complex hyperbolic geometry of the moduli space of cubic surfaces, J. Algebraic Geom. 11 (2002), no. 4, 659-724. MR 1910264 (2003m:32011)

[ACT11] , The moduli space of cubic threefolds as a ball quotient, Mem. Amer. Math. Soc. 209 (2011), no. 985, xii+70.

[AFS10] J. Alper, M. Fedorchuk, and D. I. Smyth, Singularities with $G_{m}$-action and the log minimal model program for $\bar{M}_{g}$, arXiv:1010.3751v1, 2010.

[AH12] J. Alper and D. Hyeon, GIT constructions of log canonical models of $\bar{M}_{g}$, Compact moduli spaces and vector bundles, Contemp. Math., vol. 564, Amer. Math. Soc., Providence, RI, 2012, pp. 87-106.

[AK11] M. Artebani and S. Kondō, The moduli of curves of genus six and K3 surfaces, Trans. Amer. Math. Soc. 363 (2011), no. 3, 1445-1462.

[Ale94] V. Alexeev, Boundedness and $K^{2}$ for log surfaces, Internat. J. Math. 5 (1994), no. 6, 779-810. MR 1298994 (95k:14048)

[Ale96] Moduli spaces $M_{g, n}(W)$ for surfaces, Higher-dimensional complex varieties (Trento, 1994), de Gruyter, Berlin, 1996, pp. 1-22. MR 1463171 (99b:14010)

[Ale02] Complete moduli in the presence of semiabelian group action, Ann. of Math. (2) 155 (2002), no. 3, 611-708. MR 1923963 (2003g:14059)

[Ale13] Moduli of weighted hyperplane arrangements, with applications, Notes for the May 2013 Barcelona Advanced course "Compactifying Moduli spaces", 2013. 
[All03] D. Allcock, The moduli space of cubic threefolds, J. Algebraic Geom. 12 (2003), no. 2, 201-223. MR 1949641 (2003k:14043)

[Alp08] J. Alper, Good moduli spaces for Artin stacks, to appear in Ann. Inst. Fourier (arXiv:0804.2242), 2008.

[AM99] D. Avritzer and R. Miranda, Stability of pencils of quadrics in $\mathbf{P}^{4}$, Bol. Soc. Mat. Mexicana (3) 5 (1999), no. 2, 281-300.

[AMRT10] A. Ash, D. Mumford, M. Rapoport, and Y.S. Tai, Smooth compactifications of locally symmetric varieties, second ed., Cambridge Mathematical Library, Cambridge University Press, Cambridge, 2010, With the collaboration of Peter Scholze.

[AP09] V. Alexeev and R. Pardini, Explicit compactifications of moduli spaces of Campedelli and Burniat surfaces, arXiv:0901.4431, 2009.

[BB66] W. L. Baily, Jr. and A. Borel, Compactification of arithmetic quotients of bounded symmetric domains, Ann. of Math. (2) 84 (1966), 442-528. MR 0216035 (35 \#6870)

[BCHM10] C. Birkar, P. Cascini, C. D. Hacon, and J. McKernan, Existence of minimal models for varieties of log general type, J. Amer. Math. Soc. 23 (2010), no. 2, 405-468. MR 2601039 (2011f:14023)

[Ben11] O. Benoist, Quelques espaces de modules d'intersections complètes lisses qui sont quasi-projectifs, to appear in J. Eur. Math. Soc. (arxiv:1111.1589v2), 2011.

[BG83] R. L. Bryant and P. A. Griffiths, Some observations on the infinitesimal period relations for regular threefolds with trivial canonical bundle, Arithmetic and geometry, Vol. II, Progr. Math., vol. 36, Birkhäuser Boston, Boston, MA, 1983, pp. 77-102. MR MR717607 (86a:32044)

[BHPS13] B. Bhatt, W. Ho, Z. Patakfalvi, and C. Schnell, Moduli of products of stable varieties, Compos. Math. 149 (2013), no. 12, 2036-2070.

[BKPSB98] R. E. Borcherds, L. Katzarkov, T. Pantev, and N. I. Shepherd-Barron, Families of K3 surfaces, J. Algebraic Geom. 7 (1998), no. 1, 183-193.

[Bor72] A. Borel, Some metric properties of arithmetic quotients of symmetric spaces and an extension theorem, J. Differential Geometry 6 (1972), 543-560, Collection of articles dedicated to S. S. Chern and D. C. Spencer on their sixtieth birthdays.

[Bor97] C. Borcea, K3 surfaces with involution and mirror pairs of Calabi-Yau manifolds, Mirror symmetry, II, AMS/IP Stud. Adv. Math., vol. 1, Amer. Math. Soc., Providence, RI, 1997, pp. 717-743.

[Cat84] E. H. Cattani, Mixed Hodge structures, compactifications and monodromy weight filtration, Topics in transcendental algebraic geometry (Princeton, N.J., 1981/1982), Ann. of Math. Stud., vol. 106, Princeton Univ. Press, Princeton, NJ, 1984, pp. 75100.

[CG72] C. H. Clemens and P. A. Griffiths, The intermediate Jacobian of the cubic threefold, Ann. of Math. (2) 95 (1972), 281-356.

[Cha12] F. Charles, The Tate conjecture for K3 surfaces over finite fields, arXiv:1206.4002, 2012.

[CM12] S. Casalaina-Martin, A tour of stable reduction with applications, to appear in the proceedings of the 2011 conference in honor of Joe Harris's 60th birthday (arXiv:1207.1048), 2012.

[CMGHL14] S. Casalaina-Martin, S. Grushevsky, K. Hulek, and R. Laza, Extending the Prym map to toroidal compactifications of the moduli space of abelian varieties, preprint, 2014.

[CMJL12a] S. Casalaina-Martin, D. Jensen, and R. Laza, The geometry of the ball quotient model of the moduli space of genus four curves, Compact moduli spaces and vector bundles, Contemp. Math., vol. 564, Amer. Math. Soc., 2012, pp. 107-136.

[CMJL12b] L L L canonical models and variation of GIT for genus four canonical curves, to appear in J. Algebraic Geom. (arXiv:1203.5014), 2012.

[CML09] S. Casalaina-Martin and R. Laza, The moduli space of cubic threefolds via degenerations of the intermediate Jacobian, J. Reine Angew. Math. 633 (2009), 29-65.

[CMSP03] J. A. Carlson, S. Müller-Stach, and C. Peters, Period mappings and period domains, Cambridge Studies in Advanced Mathematics, vol. 85, Cambridge University Press, Cambridge, 2003. 
[Del79] P. Deligne, Variétés de Shimura: interprétation modulaire, et techniques de construction de modèles canoniques, Automorphic forms, representations and $L$ functions, Proc. Sympos. Pure Math., XXXIII, AMS, Providence, R.I., 1979, pp. 247-289.

[DH98] I. V. Dolgachev and Y. Hu, Variation of geometric invariant theory quotients, Inst. Hautes Études Sci. Publ. Math. 87 (1998), 5-56. MR 1659282 (2000b:14060)

[DK07] I. V. Dolgachev and S. Kondō, Moduli of K3 surfaces and complex ball quotients, Arithmetic and geometry around hypergeometric functions, Progr. Math., vol. 260, Birkhäuser, Basel, 2007, pp. 43-100.

[DM69] P. Deligne and D. Mumford, The irreducibility of the space of curves of given genus, Inst. Hautes Études Sci. Publ. Math. 36 (1969), 75-109. MR 0262240 (41 \#6850)

[DM86] P. Deligne and G. D. Mostow, Monodromy of hypergeometric functions and nonlattice integral monodromy, Inst. Hautes Études Sci. Publ. Math. 63 (1986), 5-89. MR 849651 (88a:22023a)

[DM06] C. F. Doran and J. W. Morgan, Mirror symmetry and integral variations of Hodge structure underlying one-parameter families of Calabi-Yau threefolds, Mirror symmetry. V, AMS/IP Stud. Adv. Math., vol. 38, Amer. Math. Soc., Providence, RI, 2006, pp. 517-537. MR MR2282973 (2008e:14010)

[Dol03] I. V. Dolgachev, Lectures on invariant theory, London Mathematical Society Lecture Note Series, vol. 296, Cambridge University Press, Cambridge, 2003. MR 2004511 (2004g:14051)

[Don02] S. K. Donaldson, Scalar curvature and stability of toric varieties, J. Differential Geom. 62 (2002), no. 2, 289-349. MR 1988506 (2005c:32028)

[DvGK05] I. V. Dolgachev, B. van Geemen, and S. Kondō, A complex ball uniformization of the moduli space of cubic surfaces via periods of K3 surfaces, J. Reine Angew. Math. $\mathbf{5 8 8}$ (2005), 99-148.

[Far09] G. Farkas, Birational aspects of the geometry of $\bar{M}_{g}$, Surveys in differential geometry. Vol. XIV. Geometry of Riemann surfaces and their moduli spaces, Surv. Differ. Geom., vol. 14, Int. Press, Somerville, MA, 2009, pp. 57-110. MR 2655323

[Fed12] M. Fedorchuk, The final log canonical model of the moduli space of stable curves of genus 4, Int. Math. Res. Not. IMRN 24 (2012), 5650-5672. MR 3006172

[FL13] R. Friedman and R. Laza, Semialgebraic horizontal subvarieties of Calabi-Yau type, Duke Math. J. 162 (2013), no. 12, 2077-2148.

[FM83] R. Friedman and D. R. Morrison (eds.), The birational geometry of degenerations, Progress in Mathematics, vol. 29, Birkhäuser Boston, Mass., 1983.

[Fri83] R. Friedman, A degenerating family of quintic surfaces with trivial monodromy, Duke Math. J. 50 (1983), no. 1, 203-214.

[FS86] R. Friedman and R. C. Smith, Degenerations of Prym varieties and intersections of three quadrics, Invent. Math. 85 (1986), no. 3, 615-635.

[FS13a] M. Fedorchuk and D. I. Smyth, Alternate compactifications of moduli spaces of curves, Handbook of Moduli I, Advanced Lectures in Mathematics, vol. 24, Int. Press, Somerville, MA, 2013, pp. 331-413.

[FS13b] Stability of genus five canonical curves, to appear in the proceedings of the 2011 conference in honor of Joe Harris's 60th birthday (arXiv:1302.4804), 2013.

[Fuj12] O. Fujino, Semipositivity theorems for moduli problems, arXiv:1210.5784, 2012.

[Gal13] P. Gallardo, On the GIT quotient of quintic surfaces, arXiv:1310.3534, 2013.

[GGK12] M. Green, P. Griffiths, and M. Kerr, Mumford-Tate groups and domains, Annals of Mathematics Studies, vol. 183, Princeton University Press, 2012. MR 2918237

[GH12] S. Grushevsky and K. Hulek, The class of the locus of intermediate Jacobians of cubic threefolds, Invent. Math. 190 (2012), no. 1, 119-168.

[GHS07] V. A. Gritsenko, K. Hulek, and G. K. Sankaran, The Kodaira dimension of the moduli of K3 surfaces, Invent. Math. 169 (2007), no. 3, 519-567.

[GHS13] Moduli of K3 Surfaces and Irreducible Symplectic Manifolds, Handbook of Moduli I, Advanced Lectures in Mathematics, vol. 24, Int. Press, Somerville, MA, 2013 , to appear in this volume, pp. 459-526.

[Gie77a] D. Gieseker, Global moduli for surfaces of general type, Invent. Math. 43 (1977), no. 3, 233-282. MR 0498596 (58 \#16687) 
[Gie77b] On the moduli of vector bundles on an algebraic surface, Ann. of Math. (2) 106 (1977), no. 1, 45-60. MR 466475 (81h:14014)

[Hac04] P. Hacking, Compact moduli of plane curves, Duke Math. J. 124 (2004), no. 2, 213-257. MR 2078368 (2005f:14056)

[Har77] R. Hartshorne, Algebraic geometry, Graduate Texts in Mathematics, vol. 52, Springer-Verlag, New York, 1977. MR 0463157 (57 \#3116)

[Har10] , Deformation theory, Graduate Texts in Mathematics, vol. 257, Springer, New York, 2010.

[Has00] B. Hassett, Local stable reduction of plane curve singularities, J. Reine Angew. Math. $\mathbf{5 2 0}$ (2000), 169-194.

[HH09] B. Hassett and D. Hyeon, Log canonical models for the moduli space of curves: the first divisorial contraction, Trans. Amer. Math. Soc. 361 (2009), no. 8, 4471-4489. MR 2500894 (2009m:14039)

[HH13]__ Log minimal model program for the moduli space of curves: The first flip, Ann. of Math. (2) 177 (2013), 1-58.

[HKT09] P. Hacking, S. Keel, and J. Tevelev, Stable pair, tropical, and log canonical compactifications of moduli spaces of del Pezzo surfaces, Invent. Math. 178 (2009), no. 1, $173-227$.

[HL02] G. Heckman and E. Looijenga, The moduli space of rational elliptic surfaces, Algebraic geometry 2000, Azumino (Hotaka), Adv. Stud. Pure Math., vol. 36, Math. Soc. Japan, Tokyo, 2002, pp. 185-248.

[HL10] D. Hyeon and Y. Lee, Log minimal model program for the moduli space of stable curves of genus three, Math. Res. Lett. 17 (2010), no. 4, 625-636. MR 2661168

[HMX13] C. D. Hacon, J. McKernan, and C. Xu, On the birational automorphisms of varieties of general type, Ann. of Math. (2) 177 (2013), no. 3, 1077-1111.

[Huy11] D. Huybrechts, A global Torelli theorem for hyperkaehler manifolds (after Verbitsky), to appear in Seminaire Bourbaki (arXiv:1106.5573), 2011.

[Keb13] S. Kebekus, Differential forms on singular spaces, the minimal model program, and hyperbolicity of moduli stacks, Handbook of Moduli II, Advanced Lectures in Mathematics, vol. 25, Int. Press, Somerville, MA, 2013, pp. 71-113.

[Kem78] G. R. Kempf, Instability in invariant theory, Ann. of Math. (2) 108 (1978), no. 2, 299-316. MR 506989 (80c:20057)

[Kir84] F. C. Kirwan, Cohomology of quotients in symplectic and algebraic geometry, Mathematical Notes, vol. 31, Princeton University Press, Princeton, NJ, 1984. MR 766741 (86i:58050)

[Kir85] Partial desingularisations of quotients of nonsingular varieties and their Betti numbers, Ann. of Math. (2) 122 (1985), no. 1, 41-85. MR 799252 (87a:14010)

[Kir09] _ Quotients by non-reductive algebraic group actions, Moduli spaces and vector bundles, London Math. Soc. Lecture Note Ser., vol. 359, Cambridge Univ. Press, Cambridge, 2009, pp. 311-366. MR 2537073 (2011a:14092)

[KK10] J. Kollár and S. J. Kovács, Log canonical singularities are Du Bois, J. Amer. Math. Soc. 23 (2010), no. 3, 791-813.

[KL04] H. Kim and Y. Lee, Log canonical thresholds of semistable plane curves, Math. Proc. Cambridge Philos. Soc. 137 (2004), no. 2, 273-280. MR MR2090618 (2005m:14055)

[KM97] S. Keel and S. Mori, Quotients by groupoids, Ann. of Math. (2) 145 (1997), no. 1, 193-213. MR 1432041 (97m:14014)

[Kol90] J. Kollár, Projectivity of complete moduli, J. Differential Geom. 32 (1990), no. 1, 235-268. MR 1064874 (92e:14008)

[Kol96] _ Rational curves on algebraic varieties, Ergebnisse der Mathematik und ihrer Grenzgebiete. 3. Folge., vol. 32, Springer-Verlag, Berlin, 1996.

[Kol08] J. Kollár, Husks and hulls, arXiv:0805.0576, 2008.

[Kol13a] _ Moduli of varieties of general type, Handbook of Moduli II, Advanced Lectures in Mathematics, vol. 25, Int. Press, Somerville, MA, 2013, pp. 131-157.

[Kol13b] J. Kollár, Singularities of the minimal model program, Cambridge Tracts in Mathematics, vol. 200, Cambridge University Press, Cambridge, 2013.

[Kon00] S. Kondō, A complex hyperbolic structure for the moduli space of curves of genus three, J. Reine Angew. Math. 525 (2000), 219-232. MR 1780433 (2001j:14039) 
[Kon02] - The moduli space of curves of genus 4 and Deligne-Mostow's complex reflection groups, Algebraic geometry 2000, Azumino (Hotaka), Adv. Stud. Pure Math., vol. 36, Math. Soc. Japan, Tokyo, 2002, pp. 383-400. MR 1971521 (2004h:14033)

[KSB88] J. Kollár and N. I. Shepherd-Barron, Threefolds and deformations of surface singularities, Invent. Math. 91 (1988), no. 2, 299-338. MR 922803 (88m:14022)

[KU09] K. Kato and S. Usui, Classifying spaces of degenerating polarized Hodge structures, Annals of Mathematics Studies, vol. 169, Princeton University Press, Princeton, NJ, 2009

[Laz09] R. Laza, The moduli space of cubic fourfolds, J. Algebraic Geom. 18 (2009), no. 3, 511-545. MR 2496456 (2010c:14039)

[Laz10] 는 The moduli space of cubic fourfolds via the period map, Ann. of Math. (2) 172 (2010), no. 1, 673-711. MR 2680429

[Laz12] - The KSBA compactification for the moduli space of degree two K3 pairs, arXiv:1205.3144, 2012.

[Laz13] _ _ GIT and moduli with a twist, Handbook of Moduli II, Advanced Lectures in Mathematics, vol. 25, Int. Press, Somerville, MA, 2013, pp. 259-297.

[Loo03a] E. Looijenga, Compactifications defined by arrangements. I. The ball quotient case, Duke Math. J. 118 (2003), no. 1, 151-187. MR 1978885 (2004i:14042a)

[Loo03b]__ Compactifications defined by arrangements. II. Locally symmetric varieties of type IV, Duke Math. J. 119 (2003), no. 3, 527-588. MR 2003125 (2004i:14042b)

[Loo07]__ Invariants of quartic plane curves as automorphic forms, Algebraic geometry, Contemp. Math., vol. 422, Amer. Math. Soc., Providence, RI, 2007, pp. 107-120. MR 2296435 (2008b:14045)

[Loo09]_ The period map for cubic fourfolds, Invent. Math. 177 (2009), no. 1, 213233. MR 2507640 (2010h:32013)

[LS07] E. Looijenga and R. Swierstra, The period map for cubic threefolds, Compos. Math. 143 (2007), no. 4, 1037-1049. MR 2339838 (2008f:32015)

[LZ12] Z. Li and L. Zhang, Modular forms and special cubic fourfolds, arXiv:1203.1373, 2012 .

[MFK94] D. Mumford, J. Fogarty, and F. Kirwan, Geometric invariant theory, third ed., Ergebnisse der Mathematik und ihrer Grenzgebiete (2), vol. 34, Springer-Verlag, Berlin, 1994. MR 1304906 (95m:14012)

[Mil13] J.S. Milne, Shimura varieties and moduli, Handbook of Moduli II, Advanced Lectures in Mathematics, vol. 25, Int. Press, Somerville, MA, 2013, pp. 467-548.

[Mor84] D. R. Morrison, The Clemens-Schmid exact sequence and applications, Topics in transcendental algebraic geometry, Ann. of Math. Stud., vol. 106, Princeton Univ. Press, 1984, pp. 101-119.

[Mor09] I. Morrison, GIT constructions of moduli spaces of stable curves and maps, Surveys in differential geometry. Vol. XIV. Geometry of Riemann surfaces and their moduli spaces, Surv. Differ. Geom., vol. 14, Int. Press, Somerville, MA, 2009, pp. 315-369. MR 2655332

[MP07] D. Maulik and R. Pandharipande, Gromov-Witten theory and Noether-Lefschetz theory, to appear in the proceedings of the 2011 conference in honor of Joe Harris's 60th birthday (arXiv:0705.1653), 2007.

[Muk03] S. Mukai, An introduction to invariants and moduli, Cambridge Studies in Advanced Mathematics, vol. 81, Cambridge University Press, Cambridge, 2003, Translated from the 1998 and 2000 Japanese editions by W. M. Oxbury. MR 2004218 (2004g:14002)

[Mum77] D. Mumford, Stability of projective varieties, Enseignement Math. (2) 23 (1977), no. 1-2, 39-110. MR 0450272 (56 \#8568)

[Mum83]_ On the Kodaira dimension of the Siegel modular variety, Algebraic geometry-open problems (Ravello, 1982), Lecture Notes in Math., vol. 997, Springer, Berlin, 1983, pp. 348-375.

[Nam80] Y. Namikawa, Toroidal compactification of Siegel spaces, Lecture Notes in Mathematics, vol. 812, Springer, Berlin, 1980.

[New78] P. E. Newstead, Introduction to moduli problems and orbit spaces, Tata Institute of Fundamental Research Lectures on Mathematics and Physics, vol. 51, Tata Institute of Fundamental Research, Bombay, 1978. MR 546290 (81k:14002) 
[Oda12] Y. Odaka, On the GIT stability of polarized varieties - a survey, Proceegings of the 2010 Kinosaki Symposium, 2012.

[Oda13] Y. Odaka, The GIT stability of polarized varieties via discrepancy, Ann. of Math. (2) $\mathbf{1 7 7}$ (2013), 645-661.

[OSS12] Y. Odaka, C. Spotti, and S. Sun, Compact moduli spaces of del Pezzo surfaces and Kähler-Einstein metrics, arXiv:1210.0858, 2012.

[Roh09] J. C. Rohde, Cyclic coverings, Calabi-Yau manifolds and complex multiplication, Lecture Notes in Mathematics, vol. 1975, Springer-Verlag, Berlin, 2009. MR MR2510071

[Ser06] E. Sernesi, Deformations of algebraic schemes, Grundlehren der Mathematischen Wissenschaften [Fundamental Principles of Mathematical Sciences], vol. 334, Springer-Verlag, Berlin, 2006.

[Ses67] C. S. Seshadri, Space of unitary vector bundles on a compact Riemann surface, Ann. of Math. (2) 85 (1967), 303-336. MR 0233371 (38 \#1693)

[Sha79] J. Shah, Insignificant limit singularities of surfaces and their mixed Hodge structure, Ann. of Math. (2) 109 (1979), no. 3, 497-536.

[Sha80] _ A complete moduli space for K3 surfaces of degree 2, Ann. of Math. (2) 112 (1980), no. 3, 485-510. MR 595204 (82j:14030)

[Sha81a] , Degenerations of K3 surfaces of degree 4, Trans. Amer. Math. Soc. 263 (1981), no. 2, 271-308.

[Sha81b] Stability of two-dimensional local rings. I, Invent. Math. 64 (1981), no. 2, $297-343$.

[Sta13] The Stacks Project Authors (J.A. de Jong et al.), Stacks project, http://stacks.math.columbia.edu, 2013.

[Ste81] J. H. M. Steenbrink, Cohomologically insignificant degenerations, Compositio Math. 42 (1980/81), no. 3, 315-320.

[SU02] Y. Shimizu and K. Ueno, Advances in moduli theory, Translations of Mathematical Monographs, vol. 206, American Mathematical Society, 2002.

[Tai82] Y.S. Tai, On the Kodaira dimension of the moduli space of abelian varieties, Invent. Math. 68 (1982), no. 3, 425-439.

[Tha94] M. Thaddeus, Stable pairs, linear systems and the Verlinde formula, Invent. Math. 117 (1994), no. 2, 317-353. MR 1273268 (95e:14006)

[Tha96] Geometric invariant theory and flips, J. Amer. Math. Soc. 9 (1996), no. 3, 691-723. MR 1333296 (96m:14017)

[Vak06] R. Vakil, Murphy's law in algebraic geometry: badly-behaved deformation spaces, Invent. Math. 164 (2006), no. 3, 569-590.

[Vie95] E. Viehweg, Quasi-projective moduli for polarized manifolds, Ergebnisse der Mathematik und ihrer Grenzgebiete (3), vol. 30, Springer-Verlag, Berlin, 1995.

[vO05] M. A. van Opstall, Moduli of products of curves, Arch. Math. (Basel) 84 (2005), no. $2,148-154$.

[Voi86] C. Voisin, Théorème de Torelli pour les cubiques de $\mathbf{P}^{5}$, Invent. Math. 86 (1986), no. $3,577-601$.

[Voi07] Hodge theory and complex algebraic geometry. I, II, english ed., Cambridge Studies in Advanced Mathematics, vol. 76/77, Cambridge University Press, Cambridge, 2007.

[VZ05] E. Viehweg and K. Zuo, Complex multiplication, Griffiths-Yukawa couplings, and rigidity for families of hypersurfaces, J. Algebraic Geom. 14 (2005), no. 3, 481-528.

[VZ10] A. D. Vedova and F. Zudda, Scalar curvature and asymptotic Chow stability of projective bundles and blowups, arXiv:1009.5755, 2010.

[WX12] X. Wang and $\mathrm{C}$. Xu, Nonexistence of asymptotic GIT compactification, arXiv:1212.0173, 2012.

Stony Brook University, Department of Mathematics, Stony Brook, NY 11794.

E-mail address: rlaza@math.sunysb.edu

Stony Brook University, Department of Mathematics, Stony Brook, Ny 11794

E-mail address: rlaza@math.sunysb.edu 